\title{
ON THE GROWTH RATE OF A LINEAR STOCHASTIC RECURSION WITH MARKOVIAN DEPENDENCE
}

\author{
DAN PIRJOL AND LINGJIONG ZHU
}

\begin{abstract}
We consider the linear stochastic recursion $x_{i+1}=a_{i} x_{i}+b_{i}$ where the multipliers $a_{i}$ are random and have Markovian dependence given by the exponential of a standard Brownian motion and $b_{i}$ are i.i.d. positive random noise independent of $a_{i}$. Using large deviations theory we study the growth rates (Lyapunov exponents) of the positive integer moments $\lambda_{q}=\lim _{n \rightarrow \infty} \frac{1}{n} \log \mathbb{E}\left[\left(x_{n}\right)^{q}\right]$ with $q \in \mathbb{Z}_{+}$. We show that the Lyapunov exponents $\lambda_{q}$ exist, under appropriate scaling of the model parameters, and have non-analytic behavior manifested as a phase transition. We study the properties of the phase transition and the critical exponents using both analytic and numerical methods.
\end{abstract}

\section{INTRODUCTION}

Random recursions are widely used to model processes in economics, biology, computer science and physics, see e.g. [17, 18, 22, 24, 32]. The best known is the Kesten process which is defined by the linear stochastic recursion [18]

$$
x_{i+1}=a_{i} x_{i}+b_{i}
$$

where $a_{i}, b_{i}$ are random i.i.d. real variables with $a_{i}>0$. The distributional properties of this process and the conditions for the existence of stationary and ergodic distributions are well-known [18, 35, 16]. The process (1) has a unique stationary solution, provided that $\mathbb{E}\left[\log a_{i}\right]<0$ and $\mathbb{E}\left[\log \left(b_{i}\right)_{+}\right]<\infty$. Under these conditions the stationary distribution has power-like tails (more precisely of regular variation) $\mathbb{P}\left(x_{n}>X\right) \sim X^{-\mu}$ for $X \rightarrow \infty$, where the exponent $\mu$ is determined by the equation $\mathbb{E}\left[a_{i}^{\mu}\right]=1$. See [23] for a recent overview of the properties of this process.

The generalization of the process (1) to the case of random coefficients $a_{i}, b_{i}$ with Markovian dependence has been also considered in the mathematical literature [30, 31].

In this paper we consider a linear stochastic recursion of the form (1) with stochastic multipliers $a_{i}$ following a Markov process given by the exponential

Date: 10 November 2014. Revised: 11 May 2015.

2000 Mathematics Subject Classification. 60G99,60K99,82B26,60F10.

Key words and phrases. linear stochastic recursion, Lyapunov exponent, phase transitions, critical exponent, large deviations. 
of a standard Brownian motion. We consider the stochastic recursion defined by

$$
x_{i+1}=a_{i} x_{i}+b_{i}, \quad a_{i}=1+\rho e^{\sigma W_{i}-\frac{1}{2} \sigma^{2} t_{i}}
$$

with initial condition $x_{0}>0$ and $b_{i}$ are i.i.d. positive random variables independent of $\left(a_{i}\right)_{i=0}^{\infty}$. $W_{i}$ is a standard Brownian motion sampled on the uniformly spaced times $t_{i}$ with time step $t_{i+1}-t_{i}=\tau$ and $t_{0}=0$, i.e. $W_{i}=W\left(t_{i}\right)$, where $W(t)$ is a standard Brownian motion starting at 0 at time 0 . The parameters $\sigma, \rho \in \mathbb{R}_{+}$are real positive numbers. Although superficially similar to the linear recursion (1), the process (2) has a different qualitative from the Kesten process as it is non-stationary.

For the moment, we will assume the homogeneous case $b_{i} \equiv 0$, and we consider the random multiplicative process

$$
x_{i+1}=a_{i} x_{i}, \quad a_{i}=1+\rho e^{\sigma W_{i}-\frac{1}{2} \sigma^{2} t_{i}}
$$

The process (2) under the full stated assumptions will be considered in Section 10.2. The process (3) is a stochastic growth process with correlated multipliers $a_{i}>1$. It can be regarded as a discrete time version of the continuous-time process $d X_{t}=\rho e^{\sigma W_{t}-\frac{1}{2} \sigma^{2} t} X_{t} d t$ by writing it as

$$
x_{i+1}-x_{i}=\rho e^{\sigma W_{i}-\frac{1}{2} \sigma^{2} t_{i}} x_{i}
$$

The process for $X_{t}$ is solved in terms of the time integral of the geometric Brownian motion

$$
X_{t}=X_{0} \exp \left(\rho \int_{0}^{t} d s e^{\sigma W_{s}-\frac{1}{2} \sigma^{2} s}\right) .
$$

The time integral of the geometric Brownian motion appears in many problems of statistics, probability and mathematical finance, and its distributional properties have been widely studied [13, 14, 36. We will show that the discrete time version (3) of this process has new and interesting properties, which are qualitatively different from those of the continuous time case.

The process (3) appears in several mathematical finance problems. For example, it describes the bank account under discrete time compounding with interest rates following a geometric Brownian motion. This corresponds to the money market account in the Black-Derman-Toy model of stochastic interest rates [5]. This quantity plays a central role in the simulation of a short rate model, as it gives the numeraire in the risk-neutral measure. A good understanding of its distributional properties is crucial for the numerical application of the model.

The process (3) can be also related to the statistical mechanics of disordered systems, see [6] for an overview. The random variable $x_{n}$ is related to the grand partition function of a one-dimensional lattice gas of non-interacting particles placed in a random external field $\phi_{i}$ given by a 
Brownian motion. This is given by

$$
\mathcal{Z}[\phi]=\prod_{k=1}^{n}\left(1+e^{-\beta \phi_{i}+\beta \mu}\right),
$$

with $\beta=1 / T$ the inverse temperature and $\mu$ the chemical potential. The partition function $\mathcal{Z}[\phi]$ has the same general form as $x_{n}$ given by the process (3). Such systems have been studied in [7, 10, 11] on the case of a continuous one-dimensional gas in the quenched random field approximation.

We would like to study the large $n$ asymptotics of the growth rate of the positive integer moments $\mathbb{E}\left[\left(x_{n}\right)^{q}\right]$ with $q \in \mathbb{Z}_{+}$. More precisely, we will consider the $n \rightarrow \infty$ limit at fixed

$$
\beta=\frac{1}{2} \sigma^{2} t_{n} n=\frac{1}{2} \sigma^{2} \tau n^{2} .
$$

Using large deviations theory we will show that the following limit exists and defines a Lyapunov exponent for the growth rate of the expectation

$$
\lambda(\rho, \beta ; q)=\lim _{n \rightarrow \infty} \frac{1}{n} \log \mathbb{E}\left[\left(x_{n}\right)^{q}\right], \quad q \in \mathbb{Z}_{+} .
$$

The process (3) was considered in [26, where the positive integer moments $\mathbb{E}\left[\left(x_{n}\right)^{q}\right]$ have been computed exactly (but numerically) for finite $n$. This numerical study showed that these moments have a sharp explosion for sufficiently large $n$ or $\sigma$, which signals the appearance of heavy tailed distributions for the random variable $x_{n}$. This phenomenon can be studied by mapping the problem to a one-dimensional lattice gas, and the explosion is related to a gas-liquid phase transition in this system [27].

The analyticity of Lyapunov exponents has been extensively studied for products of random matrices with i.i.d. distributed matrix elements, see [9, 29]. The problem considered here is somewhat different, as the Lyapunov exponent refers to a deterministic quantity, the expectation of the random variable $x_{n}$.

The paper is organized as follows. In Section 2, we use large deviations theory to prove the existence of the Lyapunov exponent and express it as a variational problem. The convergence of the random variable $\frac{1}{n} \log x_{n}$ as $n \rightarrow \infty$ and its fluctuations are studied in Section 3. We analyze the variational problem for the Lyapunov exponent in Section 4 , and present a numerical study of its solution in Section 5. Section 6 summarizes an approximation to the solution of the variational problem which is equivalent to the mean field approximation. A rigorous study of the phase transitions is presented in Section 7. The slope of the phase transition curve and the critical exponents are studied in Sections 8 and 9, respectively. Generalizations to the Lyapunov exponents of the positive integer moments $q \geq 2$ and the linear stochastic recursion will be studied in Section 10 respectively. 


\section{Lyapunov Exponent and Large Deviations}

Let us consider the discrete time random multiplicative process defined by

$$
x_{i+1}=a_{i} x_{i}, \quad a_{i}=1+\rho e^{\sigma W_{i}-\frac{1}{2} \sigma^{2} t_{i}},
$$

where $W_{i}=W\left(t_{i}\right)$ is the value of standard Brownian motion at time $t_{i}$ and $t_{i}=i \tau$. The parameters $\rho$ and $\sigma$ are real positive constants.

Define the Lyapunov exponent $\lambda(\rho, \beta)$ as

$$
\lambda(\rho, \beta):=\lim _{n \rightarrow \infty} \frac{1}{n} \log \mathbb{E}\left[x_{n}\right]
$$

We would like to show the existence of the Lyapunov exponent and express it in terms of a variational formula. Our proof uses the large deviations theory from probability. Before we proceed, recall that a sequence $\left(P_{n}\right)_{n \in \mathbb{N}}$ of probability measures on a topological space $\mathbb{X}$ satisfies the large deviations principle with rate function $\mathcal{I}: \mathbb{X} \rightarrow \mathbb{R}$ if $\mathcal{I}$ is non-negative, lower semicontinuous and for any measurable set $A$, we have

$$
-\inf _{x \in A^{o}} \mathcal{I}(x) \leq \liminf _{n \rightarrow \infty} \frac{1}{n} \log P_{n}(A) \leq \limsup _{n \rightarrow \infty} \frac{1}{n} \log P_{n}(A) \leq-\inf _{x \in \bar{A}} \mathcal{I}(x) .
$$

Here, $A^{o}$ is the interior of $A$ and $\bar{A}$ is its closure. We refer to [12] and [34] for general background of the theory and the applications of large deviations.

Theorem 1. The limit $\lambda(\rho, \beta):=\lim _{n \rightarrow \infty} \frac{1}{n} \log \mathbb{E}\left[x_{n}\right]$ exists and it can be expressed as a variational formula

$$
\lambda(\rho, \beta)=\sup _{g \in \mathcal{G}}\left\{\log \rho g(1)+\beta \int_{0}^{1}(g(1)-g(x))^{2} d x-\int_{0}^{1} I\left(g^{\prime}(x)\right) d x\right\},
$$

where $I(x):=x \log x+(1-x) \log (1-x)$ and

$\mathcal{G}:=\left\{g:[0,1] \rightarrow[0,1]: g(0)=0, g\right.$ is absolutely continuous and $\left.0 \leq g^{\prime} \leq 1\right\}$.

Proof. By iterating the expression $x_{i+1}=a_{i} x_{i}$, we get

$$
\begin{aligned}
\mathbb{E}\left[x_{n}\right] & =\mathbb{E}\left[a_{n-1} a_{n-2} \cdots a_{1} a_{0} x_{0}\right] \\
& =x_{0} \mathbb{E}\left[\prod_{i=0}^{n-1}\left(1+\rho e^{\sigma W_{i}-\frac{1}{2} \sigma^{2} t_{i}}\right)\right] \\
& =x_{0} \mathbb{E}\left[\prod_{i=0}^{n-1}\left(1+e^{\log \rho+\sigma W_{i}-\frac{1}{2} \sigma^{2} t_{i}}\right)\right] \\
& =x_{0} 2^{n} \mathbb{E}\left[\prod_{i=0}^{n-1}\left(\frac{1}{2}+\frac{1}{2} e^{\log \rho+\sigma W_{i}-\frac{1}{2} \sigma^{2} t_{i}}\right)\right] \\
& =x_{0} 2^{n} \mathbb{E}\left[\prod_{i=0}^{n-1} e^{\left(\log \rho+\sigma W_{i}-\frac{1}{2} \sigma^{2} t_{i}\right) Y_{i}}\right],
\end{aligned}
$$


where $Y_{i}$ are i.i.d. random variables taking values 1 and 0 with probability $1 / 2$ and independent of the Brownian motion $W(t)$. Notice that $W_{i}$ are dependent random variables. However, their increments are independent. Therefore, it is natural to define their increments as $V_{i}=W_{i+1}-W_{i}, \quad i=$ $0,1,2,3, \ldots$ and therefore, we get

$$
\begin{aligned}
\mathbb{E}\left[x_{n}\right] & =x_{0} 2^{n} \mathbb{E}\left[\prod_{i=0}^{n-1} e^{\left(\log \rho+\sigma W_{i}-\frac{1}{2} \sigma^{2} t_{i}\right) Y_{i}}\right] \\
& =x_{0} 2^{n} \mathbb{E}\left[e^{\log \rho \sum_{i=0}^{n-1} Y_{i}+\sum_{i=0}^{n-1} \sum_{j=0}^{i-1}\left(\sigma V_{j}-\frac{1}{2} \sigma^{2} \tau\right) Y_{i}}\right] \\
& =x_{0} 2^{n} \mathbb{E}\left[e^{\log \rho \sum_{i=0}^{n-1} Y_{i}+\sum_{j=0}^{n-2}\left(\sum_{i=j+1}^{n-1} Y_{i}\right)\left(\sigma V_{j}-\frac{1}{2} \sigma^{2} \tau\right)}\right]
\end{aligned}
$$

It is clear that $\sigma V_{j}-\frac{1}{2} \sigma^{2} \tau$ are i.i.d. Gaussian random variables with mean $-\frac{1}{2} \sigma^{2} \tau$ and variance $\sigma^{2} \tau$. Therefore,

$$
\begin{aligned}
\mathbb{E}\left[x_{n}\right] & =x_{0} 2^{n} \mathbb{E}\left[e^{\log \rho \sum_{i=0}^{n-1} Y_{i}-\frac{1}{2} \sigma^{2} \tau \sum_{j=0}^{n-2}\left(\sum_{i=j+1}^{n-1} Y_{i}\right)+\frac{1}{2} \sigma^{2} \tau \sum_{j=0}^{n-2}\left(\sum_{i=j+1}^{n-1} Y_{i}\right)^{2}}\right] \\
& =x_{0} 2^{n} \mathbb{E}\left[e^{A_{n}}\right]
\end{aligned}
$$

where the exponent $A_{n}$ is given by

$$
\begin{aligned}
A_{n} & =\log \rho \sum_{i=1}^{n} Y_{i}+\frac{\beta}{n^{2}} \sum_{j=0}^{n-1}\left(\sum_{i=j+1}^{n} Y_{i}\right)^{2}+r_{n} \\
& =\log \rho \sum_{i=1}^{n} Y_{i}+\frac{\beta}{n} \int_{0}^{1}\left(\sum_{i=\lfloor n x\rfloor+1}^{n} Y_{i}\right)^{2} d x+r_{n} \\
& =n\left[\log \rho\left(\frac{1}{n} \sum_{i=1}^{n} Y_{i}\right)+\beta \int_{0}^{1}\left(\frac{1}{n} \sum_{i=1}^{n} Y_{i}-\frac{1}{n} \sum_{i=1}^{\lfloor n x\rfloor} Y_{i}\right)^{2} d x\right]+o(n) .
\end{aligned}
$$

The correction $r_{n}$ is bounded by a deterministic constant and is negligible in the large $n$ limit. Recalling that we are interested in the $n \rightarrow \infty$ limit at fixed $\beta=\frac{1}{2} \sigma^{2} \tau n^{2}$, it is easy to see that the middle term in 16 satisfies

$$
0 \leq \frac{1}{2} \sigma^{2} \tau \sum_{j=0}^{n-2}\left(\sum_{i=j+1}^{n-1} Y_{i}\right) \leq \frac{\beta}{n^{2}} n^{2}=\beta,
$$

and it is negligible.

By large deviations theory in probability, the Mogulskii theorem (see Theorem 5.1.2 in [12] $)$ says that $\mathbb{P}\left(\frac{1}{n} \sum_{i=1}^{\lfloor n \cdot\rfloor} Y_{i} \in \cdot\right)$ satisfies a sample path large deviations principle on the space $L_{\infty}[0,1]$ (i.e. the space of functions on $[0,1]$ equipped with supremum norm) with the rate function

$$
\int_{0}^{1} I\left(g^{\prime}(x)\right) d x
$$


where $g(0)=0, g$ is absolutely continuous, $0 \leq g^{\prime} \leq 1$, and the rate function is $+\infty$ otherwise and $I(x)=x \log x+(1-x) \log (1-x)+\log 2$ is a relative entropy function. Informally speaking, it says that

$$
\mathbb{P}\left(\frac{1}{n} \sum_{i=1}^{\lfloor n x\rfloor} Y_{i} \simeq g(x), 0 \leq x \leq 1\right) \simeq e^{-n \int_{0}^{1} I\left(g^{\prime}(x)\right) d x+o(n)},
$$

as $n \rightarrow \infty$.

In large deviations theory, the celebrated Varadhan's lemma says that if $P_{n}$ satisfies a large deviations principle with rate function $\mathcal{I}(x)$ on $\mathbb{X}$ and $F: \mathbb{X} \rightarrow \mathbb{R}$ is a bounded and continuous function, then

$$
\lim _{n \rightarrow \infty} \frac{1}{n} \log \int_{\mathbb{X}} e^{n F(x)} d P_{n}(x)=\sup _{x \in \mathbb{X}}\{F(x)-\mathcal{I}(x)\} .
$$

It is easy to check that for any $g \in L_{\infty}[0,1] \cap \mathcal{G}$,

$$
g \mapsto \log \rho \cdot g(1)+\beta \int_{0}^{1}(g(1)-g(x))^{2} d x
$$

is a bounded and continuous map.

Hence, by Varadhan's lemma, we conclude that the $\operatorname{limit}_{\lim } \rightarrow \infty \frac{1}{n} \log \mathbb{E}\left[x_{n}\right]$ exists and is given by the variational problem (12).

We present next a few immediate implications of this result for the asymptotics of the Lyapunov exponent in the limiting cases $\beta \rightarrow 0, \infty$ and $\rho \rightarrow \infty$.

Proposition 2. (i) The $\beta \rightarrow 0$ limit for the Lyapunov exponent is

$$
\lambda(\rho, 0)=\log (\rho+1)
$$

(ii) The Lyapunov exponent is bounded from above and below as

$$
\frac{1}{3} \beta+\log (1+\rho) \geq \lambda(\rho, \beta) \geq \frac{1}{3} \beta+\log \rho .
$$

(iii) These bounds give the asymptotic behavior in the large $\rho$ and large $\beta$ limits

$$
\lim _{\beta \rightarrow \infty} \frac{\lambda(\rho, \beta)}{\beta}=\frac{1}{3}
$$

and

$$
\lim _{\rho \rightarrow \infty}\left|\lambda(\rho, \beta)-\frac{\beta}{3}-\log \rho\right|=0 .
$$

Proof. i) Taking into account that $x \rightarrow I(x)$ is a convex function, Jensen's inequality implies the upper bound

$$
\begin{aligned}
\lambda(\rho, 0) & \leq \sup _{g}\left\{\log \rho g(1)-I\left(\int_{0}^{1} g^{\prime}(x) d x\right)\right\} \\
& =\sup _{g}\{\log \rho g(1)-I(g(1))\} \\
& =\sup _{0 \leq x \leq 1}\{\log \rho x-I(x)\} .
\end{aligned}
$$


On the other hand, choosing $g(x)=g(1) x$, it is clear that we have the lower bound

$$
\lambda(\rho, 0) \geq \sup _{0 \leq x \leq 1}\{x \log \rho-I(x)\} .
$$

Hence, when $\beta=0$, we must have

$$
\lambda(\rho, 0)=\sup _{0 \leq x \leq 1}\{x \log \rho-I(x)\} .
$$

At optimality, $\rho=\frac{x}{1-x}$ and therefore

$$
\lambda(\rho, 0)=\log (\rho+1) .
$$

ii) A lower bound on the Lyapunov exponent $\lambda(\rho, \beta)$ can be obtained by taking $g(x)=g(1) x$ in $(12)$, which gives

$$
\lambda(\rho, \beta) \geq \sup _{0 \leq x \leq 1}\left\{\log \rho x+\frac{\beta}{3} x^{2}-I(x)\right\} .
$$

An explicit result for this lower bound will be given below in Section 6, see Proposition 12. For now we derive a simpler but weaker lower bound by taking $g(1)=1$,

$$
\lambda(\rho, \beta) \geq \log \rho+\beta \int_{0}^{1}(1-x)^{2} d x-I(1)=\frac{\beta}{3}+\log \rho,
$$

which gives the lower bound in $(23)$.

On the other hand, by the Mean Value Theorem, $|g(1)-g(x)| \leq|1-x|$ for any $0 \leq g^{\prime} \leq 1$. Also, using the Jensen inequality for the last term as in (i), we get the upper bound

$$
\begin{aligned}
\lambda(\rho, \beta) & =\sup _{g(0)=0,0 \leq g^{\prime} \leq 1}\left\{\log \rho g(1)+\beta \int_{0}^{1}(g(1)-g(x))^{2} d x-\int_{0}^{1} I\left(g^{\prime}(x)\right) d x\right\} \\
& \leq \sup _{g(0)=0,0 \leq g^{\prime} \leq 1}\left\{\log \rho g(1)+\beta \int_{0}^{1}(1-x)^{2} d x-\int_{0}^{1} I\left(g^{\prime}(x)\right) d x\right\} \\
& =\frac{\beta}{3}+\log (\rho+1) .
\end{aligned}
$$

This proves the upper bound in (23).

Dividing by $\beta$ in (23) and taking the $\beta \rightarrow \infty$ limit we conclude that

$$
\lim _{\beta \rightarrow \infty} \frac{\lambda(\rho, \beta)}{\beta}=\frac{1}{3} .
$$

This proves the relation (24).

Subtracting $\frac{1}{3} \beta+\log \rho$ in the inequalities 23 gives the asymptotics 25

$$
\lim _{\rho \rightarrow \infty}\left|\lambda(\rho, \beta)-\frac{\beta}{3}-\log \rho\right|=0 \text {. }
$$


Remark 3. The result (i) agrees with the intuitive expectation: the limit $\beta \rightarrow 0$ corresponds to taking $\sigma \rightarrow 0$ in the random multiplicative process (9), which becomes a deterministic recursion in this limit. This is solved as $x_{n}=(1+\rho)^{n}$, and the Lyapunov exponent is given immediately by $\lambda(\rho, 0)=$ $\log (1+\rho)$.

\section{Almost Sure Limit and Fluctuations}

We prove in this section a strong Law of Large Numbers and a fluctuations result for $\frac{1}{n} \log x_{n}$. We start by considering first the simpler case of the random multiplicative model $x_{i+1}=a_{i} x_{i}$ and then we will derive the LLN under the more general assumption of the presence of i.i.d. additive noise $b_{i}$.

Proposition 4. Almost surely,

$\lim _{n \rightarrow \infty} \frac{1}{n} \log x_{n}=\lim _{n \rightarrow \infty} \frac{1}{n}\left[\log a_{n-1}+\log a_{n-2}+\cdots+\log a_{0}+\log x_{0}\right]=\log (1+\rho)$.

Proof. Let us recall that

$$
x_{n}=a_{n-1} a_{n-2} \cdots a_{1} a_{0} x_{0},
$$

where

$$
a_{i}=1+\rho e^{\sigma W_{i}-\frac{1}{2} \sigma^{2} t_{i}}
$$

where $t_{i}=i \tau$ and $\beta=\frac{1}{2} \sigma^{2} \tau n^{2}$ is a universal constant. Therefore, it is easy to check that $\frac{1}{2} \sigma^{2} t_{n}=\frac{\beta}{n} \rightarrow 0$ as $n \rightarrow \infty$.

Moreover, for any $\epsilon>0$, by Chebyshev's inequality, for any $\theta>0$,

$\mathbb{P}\left(\left|\sigma W_{n}\right| \geq \epsilon\right)=2 \mathbb{P}\left(\sigma W_{n} \geq \epsilon\right) \leq 2 \mathbb{E}\left[e^{\theta \sigma W_{n}}\right] e^{-\theta \epsilon}=2 e^{\frac{1}{2} \theta^{2} \sigma^{2} \tau n} e^{-\theta \epsilon}=2 e^{\theta^{2} \frac{\beta}{n}} e^{-\theta \epsilon}$.

By choosing $\theta=\sqrt{n}$, we get

$$
\mathbb{P}\left(\left|\sigma W_{n}\right| \geq \epsilon\right) \leq 2 e^{\beta} e^{-\sqrt{n} \epsilon},
$$

and hence $\sum_{n=1}^{\infty} \mathbb{P}\left(\left|\sigma W_{n}\right| \geq \epsilon\right)<\infty$ for any $\epsilon>0$. By Borel-Cantelli lemma, $\sigma W_{n} \rightarrow 0$ almost surely. Therefore, $a_{n} \rightarrow 1+\rho$ as $n \rightarrow \infty$ almost surely.

The main LLN result follows.

Theorem 5. Assume that $\mathbb{E}\left[b_{0}\right]<\infty$. Then,

$$
\lim _{n \rightarrow \infty} \frac{1}{n} \log x_{n}=\log (1+\rho),
$$

almost surely as $n \rightarrow \infty$.

Proof. Since $x_{n} \geq x_{0} \prod_{i=0}^{n-1} a_{i}$, by Proposition 4 .

$$
\liminf _{n \rightarrow \infty} \frac{1}{n} \log x_{n} \geq \log (1+\rho),
$$


almost surely as $n \rightarrow \infty$. On the other hand,

$$
x_{n} \leq\left(x_{0}+b_{0}+b_{1}+\cdots+b_{n-1}\right) \prod_{i=0}^{n-1} a_{i} .
$$

Therefore,

(43) $\frac{1}{n} \log x_{n} \leq \frac{1}{n} \log n+\frac{1}{n} \log \left(\frac{x_{0}}{n}+\frac{b_{0}+b_{1}+\cdots+b_{n-1}}{n}\right)+\frac{1}{n} \log \sum_{i=0}^{n-1} a_{i}$.

Since $\mathbb{E}\left[b_{0}\right]<\infty$, by strong law of large numbers, $\frac{b_{0}+b_{1}+\cdots+b_{n-1}}{n} \rightarrow \mathbb{E}\left[b_{0}\right]$ almost surely as $n \rightarrow \infty$. By Proposition 4 , we conclude that

$$
\limsup _{n \rightarrow \infty} \frac{1}{n} \log x_{n} \leq \log (1+\rho),
$$

almost surely as $n \rightarrow \infty$.

Remark 6. First, we notice that the almost sure limit $\lim _{n \rightarrow \infty} \frac{1}{n} \log x_{n}=$ $\log (1+\rho)$ is analytic everywhere in the phase plane $(\rho, \beta)$ while the Lyapunov exponent exhibits phase transitions. The almost sure limit has very different behavior than the Lyapunov exponent $\lim _{n \rightarrow \infty} \frac{1}{n} \log \mathbb{E}\left[x_{n}\right]$. The difference arises from the fact that the almost sure limit is determined by the typical events, i.e. the law of large numbers, while the Lyapunov exponent is determined by the rare events, i.e. the large deviations.

Next we present a fluctuation result.

Proposition 7. When $b_{i} \equiv 0$,

$$
\frac{\log x_{n}-n \log (1+\rho)}{\sqrt{n}} \rightarrow N\left(0, \frac{2 \beta}{3} \frac{\rho^{2}}{(1+\rho)^{2}}\right),
$$

in distribution as $n \rightarrow \infty$.

Proof. When $b_{i} \equiv 0, x_{n}=x_{0} \prod_{i=0}^{n-1} a_{i}$ and $\frac{1}{n} \log x_{n} \rightarrow \log (1+\rho)$ a.s. as $n \rightarrow \infty$. It is straightforward to compute that

$$
\begin{aligned}
\frac{\log x_{n}-n \log (1+\rho)}{\sqrt{n}} & =\frac{\log x_{0}}{\sqrt{n}}+\frac{1}{\sqrt{n}} \sum_{i=0}^{n-1} \log \left(1+\frac{\rho\left(e^{\sigma W_{i}-\frac{1}{2} \sigma^{2} t_{i}}-1\right)}{1+\rho}\right) \\
& =\frac{\log x_{0}}{\sqrt{n}}+\frac{1}{\sqrt{n}} \sum_{i=0}^{n-1} \frac{\rho}{1+\rho} \sigma W_{i}+\epsilon_{n},
\end{aligned}
$$

where

$$
\epsilon_{n}:=\frac{1}{\sqrt{n}} \sum_{i=0}^{n-1}\left[\log \left(1+\frac{\rho\left(e^{\sigma W_{i}-\frac{1}{2} \sigma^{2} t_{i}}-1\right)}{1+\rho}\right)-\frac{\rho}{1+\rho} \sigma W_{i}\right] .
$$


For any $x>-1, \log (1+x) \leq x$. Thus,

$$
\begin{aligned}
\epsilon_{n} & \leq \frac{1}{\sqrt{n}} \sum_{i=0}^{n-1}\left[\frac{\rho\left(e^{\sigma W_{i}-\frac{1}{2} \sigma^{2} t_{i}}-1\right)}{1+\rho}-\frac{\rho}{1+\rho} \sigma W_{i}\right] \\
& \leq \frac{1}{\sqrt{n}} \frac{\rho}{1+\rho} \sum_{i=0}^{n-1}\left[e^{\sigma W_{i}}-1-\sigma W_{i}\right]=: \bar{\epsilon}_{n} .
\end{aligned}
$$

Note that $\bar{\epsilon}_{n} \geq 0$ and

$$
\begin{aligned}
\mathbb{E}\left[\bar{\epsilon}_{n}\right] & =\frac{1}{\sqrt{n}} \frac{\rho}{1+\rho} \sum_{i=0}^{n-1}\left[e^{\frac{1}{2} \sigma^{2} i \tau}-1\right] \\
& =\frac{1}{\sqrt{n}} \frac{\rho}{1+\rho} \sum_{i=0}^{n-1}\left[e^{\frac{\beta i}{n^{2}}}-1\right] \\
& =\frac{1}{\sqrt{n}} \frac{\rho}{1+\rho}\left[\frac{e^{\frac{\beta}{n}}-1}{e^{\frac{\beta}{n^{2}}}-1}-n\right] \rightarrow 0,
\end{aligned}
$$

as $n \rightarrow \infty$. Thus $\bar{\epsilon}_{n} \rightarrow 0$ in probability.

For any $x \in \mathbb{R}$, let $F(x):=\log \left(1+\frac{\rho\left(e^{x}-1\right)}{1+\rho}\right)-\frac{\rho}{1+\rho} x$. Then, $F(0)=0$,

$$
\begin{aligned}
& F^{\prime}(x)=\frac{\frac{\rho}{1+\rho} e^{x}}{1+\frac{\rho}{1+\rho}\left(e^{x}-1\right)}-\frac{\rho}{1+\rho}, \\
& F^{\prime \prime}(x)=\frac{\frac{\rho}{(1+\rho)^{2}} e^{x}}{\left(1+\frac{\rho}{1+\rho}\left(e^{x}-1\right)\right)^{2}} .
\end{aligned}
$$

Thus $F^{\prime \prime}(x)>0$ for any $x \in \mathbb{R}$ and hence $F^{\prime}(x)$ is increasing. Note that $F^{\prime}(0)=0$, thus $F^{\prime}(x)<0$ for any $x<0$ and $F^{\prime}(x)>0$ for any $x>0$. Since $F(0)=0$, we conclude that $F(x) \geq 0$ for any $x \in \mathbb{R}$. Therefore,

$$
\epsilon_{n} \geq \frac{1}{\sqrt{n}} \sum_{i=0}^{n-1}\left[\sigma W_{i}-\frac{1}{2} \sigma^{2} t_{i}-\sigma W_{i}\right]=\frac{-1}{\sqrt{n}} \sum_{i=0}^{n-1} \frac{\beta i}{n^{2}} \rightarrow 0
$$

as $n \rightarrow \infty$. Hence, we conclude that $\epsilon_{n} \rightarrow 0$ in probability as $n \rightarrow \infty$. Also, we have $\frac{\log x_{0}}{\sqrt{n}} \rightarrow 0$ as $n \rightarrow \infty$. Finally, notice that $\frac{1}{\sqrt{n}} \sum_{i=0}^{n-1} \frac{\rho}{1+\rho} \sigma W_{i}$ is a normal random variable with mean zero and variance

$$
\begin{aligned}
\operatorname{Var}\left[\frac{1}{\sqrt{n}} \sum_{i=0}^{n-1} \frac{\rho}{1+\rho} \sigma W_{i}\right] & =\operatorname{Var}\left[\frac{1}{\sqrt{n}} \sum_{i=1}^{n-1} \frac{\rho}{1+\rho} \sigma(n-i)\left(W_{i}-W_{i-1}\right)\right] \\
& =\frac{1}{n} \sum_{i=1}^{n-1} \frac{\rho^{2}}{(1+\rho)^{2}} \frac{2 \beta}{n^{2}}(n-i)^{2} \\
& \rightarrow \frac{2 \beta}{3} \frac{\rho^{2}}{(1+\rho)^{2}},
\end{aligned}
$$

as $n \rightarrow \infty$. 
Proposition 8. Assume $b_{i}$ are i.i.d. non-negative random variables with finite mean.

$$
\frac{\log x_{n}-n \log (1+\rho)}{\sqrt{n}} \rightarrow N\left(0, \frac{2 \beta}{3} \frac{\rho^{2}}{(1+\rho)^{2}}\right)
$$

in distribution as $n \rightarrow \infty$.

Proof. Note that

$$
x_{n}=x_{0} \prod_{i=0}^{n-1} a_{i}+b_{0} \prod_{i=1}^{n-1} a_{i}+b_{1} \prod_{i=2}^{n-1} a_{i}+\cdots+b_{n-2} a_{n-1}+b_{n-1} .
$$

Since $a_{i} \geq 1$ and $b_{i} \geq 0$, we have $x_{n} \geq x_{0} \prod_{i=0}^{n-1} a_{i}$ and

$$
x_{n} \leq\left(x_{0}+b_{0}+b_{1}+\cdots+b_{n-1}\right) \prod_{i=0}^{n-1} a_{i}
$$

By strong law of large numbers, $\frac{1}{n}\left(x_{0}+b_{0}+b_{1}+\cdots+b_{n-1}\right) \rightarrow \mathbb{E}\left[b_{0}\right]$ a.s. as $n \rightarrow \infty$. Thus, $\frac{1}{\sqrt{n}} \log \left(x_{0}+b_{0}+b_{1}+\cdots+b_{n-1}\right) \rightarrow 0$ a.s. as $n \rightarrow \infty$. The result then follows from Proposition 7 .

\section{The Variational Problem}

We give in this Section the solution of the variational problem in Theorem 1 for the Lyapunov exponent $\lambda(\rho, \beta)$. The variational problem in 12 can be formulated equivalently in terms of the function $f(x)=g^{\prime}(x)$ and the functional $\Lambda[f]$ defined as

$$
\begin{aligned}
& \lambda(\rho, \beta)=\sup _{f(x)} \Lambda[f] \\
& \Lambda[f] \equiv \log \rho \int_{0}^{1} d x f(x)+\beta \int_{0}^{1} d x\left(\int_{x}^{1} d y f(y)\right)^{2}-\int_{0}^{1} d x I(f(x))
\end{aligned}
$$

defined in terms of a function $f:[0,1] \rightarrow[0,1]$ subject to the constraints

$$
0 \leq f(x) \leq 1 \text {. }
$$

The functional $\Lambda[f]$ defined in $(56)$ can be written in a more symmetrical form as

$$
\begin{aligned}
\Lambda[f] & =\log \rho \int_{0}^{1} d x f(x)+\beta \int_{0}^{1} d x\left(\int_{x}^{1} d y f(y)\right)^{2}-\int_{0}^{1} d x I(f(x)) \\
& =\int_{0}^{1} d x\left\{\log \rho f(x)+\beta \int_{0}^{1} d y \int_{0}^{1} d z K(z, y) f(z) f(y)-I(f(x))\right\},
\end{aligned}
$$

where the kernel $K(z, y)$ is $K(z, y)=\min (z, y)$.

Taking the functional derivative of $(58)$ with respect to $f$ we get the Euler-Lagrange equation

$$
\frac{\delta \Lambda[f]}{\delta f}=\log \rho+2 \beta \int_{0}^{1} d z K(y, z) f(z)-\log \frac{f(y)}{1-f(y)}=0 .
$$


This integral equation can be transformed into a differential equation by writing out the integral over the kernel in an explicit form

$$
\log \rho+2 \beta \int_{0}^{y} z f(z) d z+2 \beta y \int_{y}^{1} f(z) d z-\log \frac{f(y)}{1-f(y)}=0 .
$$

Take one derivative with respect to $y$

$$
2 \beta \int_{y}^{1} d z f(z)=\frac{d}{d y} \log \frac{f(y)}{1-f(y)}=\frac{1}{f(y)(1-f(y))} f^{\prime}(y) .
$$

Taking another derivative with respect to $y$ we obtain finally a second order differential equation for the optimizer function $f(y)$

$$
2 \beta f(y)=-\frac{d^{2}}{d y^{2}} \log \frac{f(y)}{1-f(y)} .
$$

This must be solved with the boundary conditions

$$
f(0)=\frac{\rho}{1+\rho}, \quad f^{\prime}(1)=0 .
$$

The first boundary condition (at $y=0$ ) is obtained by taking $y=0$ in the Euler-Lagrange equation (59). The integral vanishes and we get an equation for $f(0)$ which is solved with the result shown above. The second boundary condition (at $y=1$ ) is obtained by taking $y=1$ in equation (61).

Remark 9. The functional $\Lambda[f]$ given in (58) has a simple physical interpretation: this is related to the Landau potential (grand potential) $\Omega$ of a gas with density $f(y)$ enclosed in a box $(0,1)$. The particles of the gas interact by an attractive 2-body interaction with potential $-2 K(z, y)$. The gas is in contact with a thermostat of temperature $T=1 / \beta$ and a reservoir of particles with chemical potential $\mu=T \log \rho$.

This can be seen by writing the extremal value of the functional (58) as

$$
\lambda(\rho, \beta)=-\frac{1}{T} \Omega=-\frac{1}{T}(U-T S-\mu N)
$$

with $N$ the total particle number, $S$ the entropy and $U$ the energy

$$
\begin{aligned}
N & =\int_{0}^{1} d x f(x) \\
S & =-\int_{0}^{1} d x I(f(x)) \\
U & =-\int_{0}^{1} d y d z K(y, z) f(y) f(z)
\end{aligned}
$$

and $f(x)$ is given by the solution of the Euler-Lagrange (59). The equation for the density $f(x)$ is an analog of the isothermal Lane-Emden equation discussed in the canonical ensemble in [19]. 
4.1. Solution of the Euler-Lagrange equation. The equation (62) can be alternatively expressed in terms of the function

$$
h(y)=\log \frac{f(y)}{1-f(y)} .
$$

The function $h(y)$ satisfies

$$
h^{\prime \prime}(y)=-2 \beta \frac{e^{h(y)}}{1+e^{h(y)}}
$$

with boundary conditions

$$
h(0)=\log \rho, \quad h^{\prime}(1)=0 .
$$

The solution of this equation is presented in the Appendix A. Furthermore, it can be shown that the functional $\Lambda[h]$ depends only on $h(1)$, and is given explicitly by the following Proposition.

Proposition 10. If $f(x)$ is a solution of the Euler-Lagrange equation (59), then $\Lambda[f]$ depends only on $f(1)$ or equivalently $h(1)$. This is given by the relation

$$
\Lambda[f]=\log \left(1+e^{h(1)}\right)-\frac{1}{\sqrt{\beta}} \int_{\log \rho}^{h(1)} d x \sqrt{\log \frac{1+e^{h(1)}}{1+e^{x}}} .
$$

Proof. The proof is given in the Appendix A.

In conclusion, the Lyapunov exponent is given by

$$
\lambda(\rho, \beta)=\sup _{h(1)} \Lambda[h(1)] .
$$

The condition $\frac{d \Lambda[x]}{d x}=0$ gives an equation for $h(1)$

$$
F(h(1) ; \rho)=2 \sqrt{\beta}
$$

where $F(a ; \rho)$ is given by

$$
F(a ; \rho) \equiv \int_{\log \rho}^{a} \frac{d x}{\sqrt{\log \frac{1+e^{a}}{1+e^{x}}}} .
$$

The variational problem is now solved in 2 steps.

1. For given $(\rho, \beta)$, find $h(1)$ by solving the equation $(73)$. This could have one or three solutions, depending on the values of $\rho, \beta$.

2. For each solution for $h(1)$ obtained in the previous step, compute the functional $\Lambda[f]$ using equation (71). Take the supremum over these values. This gives the Lyapunov exponent $\lambda(\rho, \beta)$.

From this solution one can see that the Lyapunov exponent $\lambda(\rho, \beta)$ is a continuous function of its arguments, as it is the supremum of a family of continuous functions, depending in a continuous way on the parameters $\rho, \beta$. 


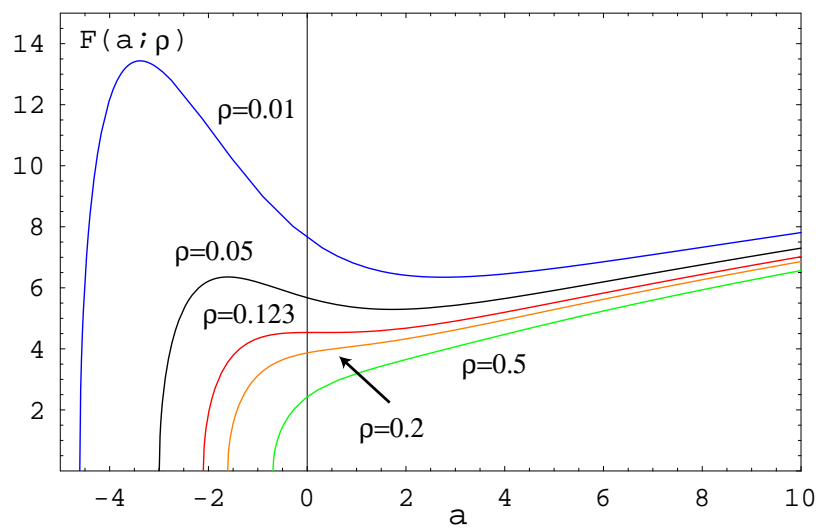

Figure 1. The function $F(a ; \rho)$ vs $a$ for several values of $\rho$ as shown.

\section{Numerical STUDY OF THE SOLUtion}

We study in this section the numerical solution of the variational problem described in the previous section, and present the results for the Lyapunov exponent. The first step of the solution consists in finding $h(1)$ from the solution of the equation $(73)$. We show in Figure 1 plots of the function $F(a ; \rho)$ for several values of $\rho=0.01,0.05,0.123,0.2,0.5$. The intersection of the curve $F(a ; \rho)$ with the horizontal line $2 \sqrt{\beta}$ determines $a=h(1)$ for given $\beta$.

We note from Figure 1 that the shape of $F(a ; \rho)$ is qualitatively different for $\rho$ above or below a certain critical value $\rho_{c} \simeq 0.12$. For $\rho>\rho_{c}$ the function $F(a ; \rho)$ is strictly increasing, while for $\rho<\rho_{c}$ it has a minimum and a maximum. At $\rho=\rho_{c}$ the function $F\left(a ; \rho_{c}\right)$ has an inflection point. If $\rho>\rho_{c}$ the equation (73) has a unique solution for $h(1)$, while for $\rho<\rho_{c}$ this equation has one or three solutions, depending on $\beta$.

The Lyapunov exponent $\lambda(\rho, \beta)$ is given by the supremum of the functional $\Lambda[h(1)]$ given in 21 over the solutions of the equation $F(a ; \rho)=2 \sqrt{\beta}$. The functional $\Lambda[h(1)]$ takes a simpler form if we choose as independent variable the integral $I_{0}$ instead of $h(1)$. These two variables are related by

$$
d \equiv \int_{0}^{1} d x f(x)=\frac{(1+\rho)^{2}}{2 \beta \rho} f^{\prime}(0)=\frac{1}{2 \beta} h^{\prime}(0)=\frac{1}{\sqrt{\beta}} \sqrt{\log \frac{1+e^{h(1)}}{1+\rho}} .
$$

The variable $d \equiv I_{0}$ takes values in $d \in(0,1)$.

We have

$$
\Lambda(d)=\beta d^{2}+\log (1+\rho)-2 \beta(1+\rho) d^{3} \int_{0}^{1} \frac{y^{2} d y}{1+\rho-e^{\beta d^{2}\left(y^{2}-1\right)}} .
$$

We will use this expression for the numerical evaluation of the Lyapunov exponent $\lambda(\rho, \beta)=\sup _{d \in(0,1)} \Lambda(d)$. 
We present in Figure 2 plots of the Lyapunov exponent $\lambda(\rho, \beta)=\sup _{d} \Lambda(d)$ These plots show $\lambda(\rho, \beta)$ vs $\beta$ at fixed $\rho=0.025,0.05,0.125,0.2$. The Lyapunov exponent has a discontinuous derivative at $\beta_{\mathrm{cr}}(\rho)$ for $\rho<\rho_{c}$. The phase transition curve $\beta_{\mathrm{cr}}(\rho)$ is shown in Figure 4 as the black solid curve. It ends at the critical point $C$ with coordinates

$$
\rho_{c}=0.12328, \quad \beta_{c}=5.12013, \quad d_{c}=0.372 .
$$

Next we study the dependence of the parameter $d$ on $\rho$ and $\beta$. Start by keeping $\rho$ fixed and consider the dependence of $d$ on $\beta$. We have $\lim _{\beta \rightarrow \infty} d=$ 1 , as this value maximizes the second term in the functional $\Lambda[f]$. and $\lim _{\beta \rightarrow 0} d=\frac{\rho}{1+\rho}$, as this value maximizes the sum of the first and last terms in $\Lambda[f]$. For intermediate values of $\beta$, provided that $\rho<\rho_{c}$, $d$ has a jump discontinuity at a certain value $\beta_{\mathrm{cr}}(\rho)$ and jumps from a value $d_{2}$ to $d_{1}$. If $\rho>\rho_{c}, d$ is a continuous function of $\beta$. For $\rho=\rho_{c}, d$ is continuous but its derivative with respect to $\beta$ becomes infinite at the $\beta=\beta_{c}$ point. This behavior is shown in Fig. 3, which shows plots of $d=d(\rho, \beta)$ vs $1 / \beta$ for several values of $\rho$. The dashed curves show also $d_{1}, d_{2}$ vs $1 / \beta$ along the phase transition curve.

A similar picture holds for the dependence of $d$ on $\rho$ at fixed $\beta$. If $\beta<\beta_{c}$, $d$ is a continuous function of $\rho$. If $\beta>\beta_{c}$ it has a discontinuity at some value $\rho_{\mathrm{cr}}(\beta)$, and if $\beta=\beta_{c}$ its derivative at $\rho=\rho_{c}$ is infinite.

In Appendix $\mathrm{B}$ we derive an analytical approximation for the functional $\Lambda(d)$ in the limit $\beta d^{2} \gg 1$, which is used to obtain the properties of the phase transition curve and of $\left(d_{1}, d_{2}\right)$ along the phase transition curve in the $\beta \rightarrow \infty$ limit. This is summarized by the following result.

Proposition 11. The solutions of the variational problem for $\Lambda(d)$ given in Eq. (76) approach the following limits for very large $\beta(\rho \ll 1)$

$$
\lim _{\beta \rightarrow \infty} d_{1}=0, \quad \lim _{\beta \rightarrow \infty} d_{2}=\frac{3}{4} .
$$

The phase transition curve is given in the same limit by

$$
\beta_{\mathrm{cr}}(\rho)=-\frac{8}{3} \log \left(\frac{\rho}{1+\rho}\right) \text { as } \rho \rightarrow 0 .
$$

See Appendix B for the proof.

We comment on the relation of these results to the statistical mechanical interpretation of the variational problem. As mentioned in the Remark 9 , the Lyapunov exponent $\lambda(\rho, \beta)$ is related to the equilibrium value of the thermodynamical potential $\Omega(T, \mu)$ of a gas of particles interacting by the two-body potential $V(x, y)=-2 \min (x, y)$. The gas is enclosed in a box $x \in(0,1)$ and is maintained at fixed temperature $T=1 / \beta$ and chemical potential $\mu=T \log \rho$, which corresponds to the grand canonical ensemble. The solution of the variational problem discussed here demonstrates the presence of a phase transition in this system. The equilibrium density of the gas is given by $f(x)$. Both the distribution density $f(x)$ and its average value $d$ are discontinuous across the phase transition curve $\beta_{\mathrm{cr}}(\rho)$. 


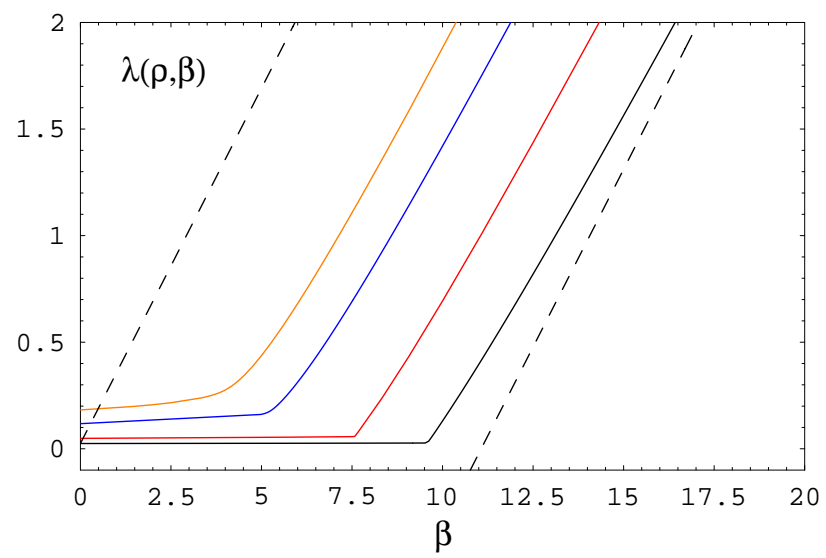

Figure 2. The Lyapunov exponent $\lambda(\rho, \beta)$ vs $\beta$ at fixed $\rho=$ 0.025 (black), $\rho=0.05$ (red), $\rho=0.125$ (blue) and $\rho=0.2$ (orange). The partial derivative $\partial_{\beta} \lambda(\rho, \beta)$ is discontinuous at one point $\beta_{\mathrm{cr}}(\rho)$ for $\rho<\rho_{c}$. The dashed curves show the upper and lower bounds on $\lambda(\rho, \beta)$ for $\rho=0.025$ given in (23). The upper bound is saturated at $\beta=0$ as shown in Proposition 2 (i).

This system is similar to the lattice gas considered in [27]. This paper considered a lattice gas with $n$ sites with interaction energy $V(x, y)=$ $-\frac{2}{n} K(x, y)$ (after appropriate rescaling of the lattice volume to 1 ). The thermodynamical properties of this system have been computed in the thermodynamical limit $n \rightarrow \infty$ in the isobaric-isothermal ensemble. The results obtained in [27] for the equation of state and the phase transition curve are in agreement with those obtained here directly in the grand canonical ensemble.

\section{Mean-Field Approximation}

A simple approximation to the solution of the variational problem is obtained by assuming that the function $f(x) \equiv a$ is a constant. This corresponds to finding the supremum over $a \in(0,1)$ of the function

$$
\bar{\lambda}(\rho, \beta)=\sup _{a \in(0,1)}\left\{a \log \rho+\frac{1}{3} \beta a^{2}-I(a)\right\} .
$$

$\bar{\lambda}(\rho, \beta)$ gives a lower bound for the exact rate function in 56

$$
\lambda(\rho, \beta) \geq \bar{\lambda}(\rho, \beta) .
$$

This approximation leads to the well-known Curie-Weiss mean field theory [17, 33, 15. This formula also appears as the limiting free energy of an edge and 2-star model in the exponential random graph models whose properties have been studied rigorously in [8, 28, 3]. For the problem considered 


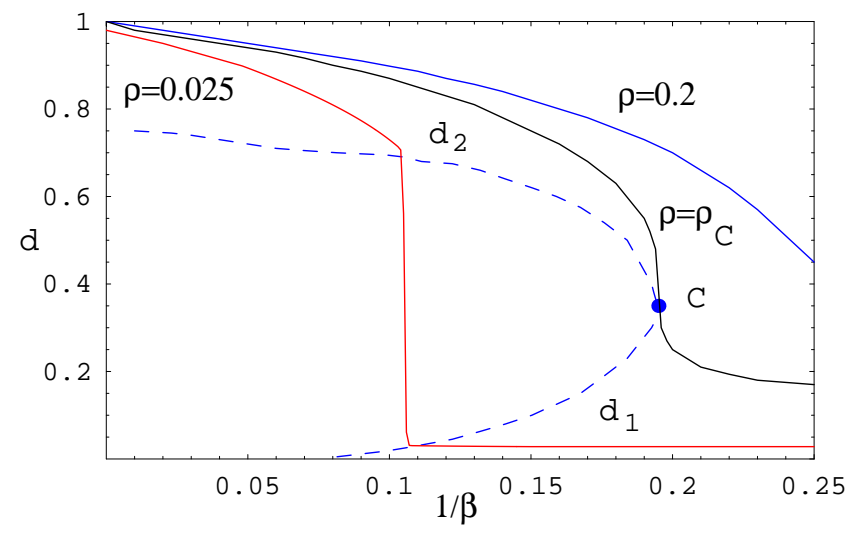

Figure 3. Plots of $d=d(\rho, \beta)$ vs $1 / \beta$ at fixed $\rho=0.025$ (red), $\rho=\rho_{c}$ (black) and $\rho=0.2$ (blue). The dashed blue curves show the values of the optimizer variable $d_{1}, d_{2}$ vs $1 / \beta$ along the phase transition curve. They become equal at the critical point $C$.

here the lower bound $\bar{\lambda}(\rho, \beta)$ gives a van der Waals approximation for the Lyapunov exponent following from the equation of state of the equivalent lattice gas with temperature $1 / \beta$ and fugacity $\rho$ [27].

We summarize below without proof the main results of the mean field approximation.

Proposition 12. i) The function $\bar{\lambda}(\rho, \beta)$ is given by

$$
\bar{\lambda}(\rho, \beta)=-\frac{1}{3} \beta a_{*}^{2}-\log \left(1-a_{*}\right)
$$

where $a_{*}=a_{*}(\rho, \beta)$ is given by

$$
a_{*}(\rho, \beta)= \begin{cases}a_{2}(\rho, \beta), & \log \rho>-\frac{1}{3} \beta \\ a_{1}(\rho, \beta), & \log \rho<-\frac{1}{3} \beta\end{cases}
$$

where $a_{1,2}$ are the smallest and largest solutions of the equation

$$
\log \rho=-\frac{2}{3} \beta a+\log \frac{a}{1-a}
$$

if this equation has multiple solutions, or the unique solution of this equation if it has only one solution.

ii) The function $\bar{\lambda}(\rho, \beta)$ has discontinuous partial derivatives with respect to its arguments at points on the phase transition curve

$$
\log \rho+\frac{1}{3} \beta=0
$$

with $\beta>\beta_{c}=6$. This curve ends at the critical point $\left(\rho_{c}, \beta_{c}\right)=\left(e^{-2}, 6\right)$. Across this curve, the solution $a_{*}$ jumps between $a_{* 1,2}=\frac{1}{2}(1 \mp \Delta(\beta))$ where $\Delta(\beta)$ is the positive non-zero solution of the nonlinear equation $\Delta=\tanh \left(\frac{1}{6} \beta \Delta\right)$. 


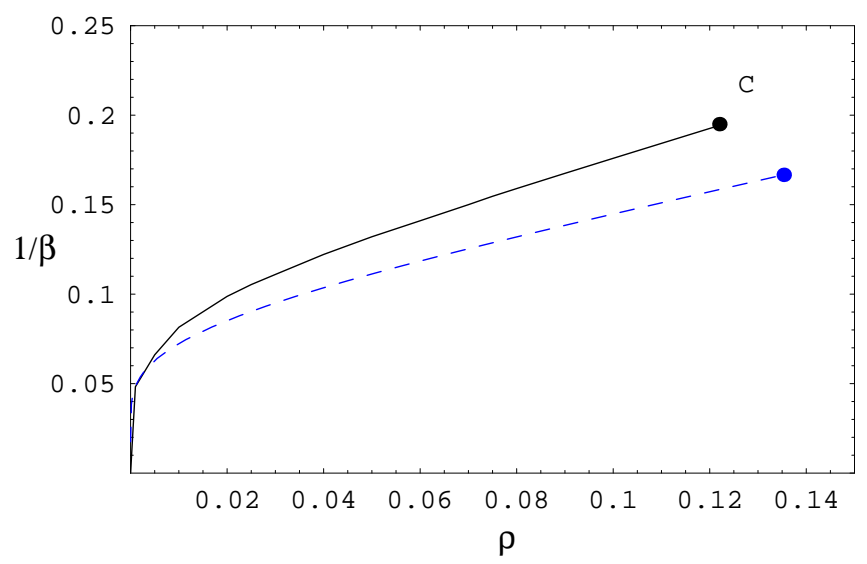

FiguRE 4. The phase transition curve $\beta_{\mathrm{cr}}(\rho)$ in coordinates $(\rho, 1 / \beta)$, obtained from the numerical solution of the model (black curve). It ends at the critical point $C$. Blue dashed curve: the phase transition curve in the mean-field approximation, which is given by Eq. (85).

Remark 13. The function $\bar{\lambda}(\rho, \beta)$ is related as $\bar{\lambda}(\rho, \beta)=\beta p_{\mathrm{vdW}}$ to the pressure of a van der Waals lattice gas with uniform long-range interaction $\varepsilon_{i j}=-\frac{2}{3 n}$ [27]. This is identical to the equation of state of a lattice gas with Kac interaction [21].

The partial derivatives of $\bar{\lambda}(\rho, \beta)$ with respect to its arguments are discontinuous across the phase transition curve. The discontinuity approaches zero near the critical point. The law of the discontinuity near the critical point is usually parameterized in terms of a critical exponent. Consider any quantity $M$ which is discontinuous across the phase transition curve. If the jump discontinuity approaches zero as $\beta \rightarrow \beta_{c}$ as

$$
\Delta M \simeq \gamma\left|\beta-\beta_{c}\right|^{\alpha}
$$

then $\alpha$ is called the critical exponent of the quantity $M$.

The jump discontinuity of the solution $a$ of the variational problem 80 for $G(a ; \rho, \beta)$ is given by the following well-known result of mean-field theory [17, 33, 15].

Proposition 14. Along the phase transition curve, very close to the critical point $\beta_{c}$, the difference of the two solutions $a_{1} \leq a_{2}$ of the variational problem (80) approaches zero as $\beta \rightarrow \beta_{c}$ as

$$
a_{2}(\beta)-a_{1}(\beta)=\Delta(\beta) \simeq \sqrt{3}\left(\frac{\beta}{\beta_{c}}-1\right)^{\frac{1}{2}}
$$


Consider the jump of the partial derivatives of $\bar{\lambda}(\rho, \beta)$ with respect to $\rho$ and $\beta$ separately. Off the phase transition curve we have

$$
\left(\frac{\partial \bar{\lambda}(\rho, \beta)}{\partial \rho}\right)_{\beta}=\frac{1}{\rho} a,
$$

which means that this derivative has a jump discontinuity across the phase transition curve

$$
\Delta\left(\frac{\partial \bar{\lambda}(\rho, \beta)}{\partial \rho}\right)_{\beta}=\frac{1}{\rho}\left(a_{2}(\beta)-a_{1}(\beta)\right)=\frac{1}{\rho} \Delta(\beta) \sim \frac{\sqrt{3}}{\rho}\left(\frac{\beta}{\beta_{c}}-1\right)^{1 / 2} .
$$

In a similar way, we have off the phase transition curve

$$
\left(\frac{\partial \bar{\lambda}(\rho, \beta)}{\partial \beta}\right)_{\rho}=\frac{1}{3} a^{2}
$$

which gives the following result for the jump of this partial derivative upon crossing the phase transition curve

$$
\Delta\left(\frac{\partial \bar{\lambda}(\rho, \beta)}{\partial \beta}\right)_{\rho}=\frac{1}{3}\left(a_{2}^{2}(\beta)-a_{1}^{2}(\beta)\right)=\frac{1}{3} \Delta(\beta) \sim \frac{1}{\sqrt{3}}\left(\frac{\beta}{\beta_{c}}-1\right)^{1 / 2}
$$

\section{Phase Transitions}

In this section, we study rigorously the phase transitions for the Lypapunov exponent $\lambda(\rho, \beta)$. Before we proceed, let us introduce the formal definition of phase transitions. We will adopt the following definition of phase and phase transition [15, 28].

Definition 15. A phase is a connected region of the parameter space $\{(\rho, \beta)\}$, maximal for the condition that the Lyapunov exponent $\lambda(\rho, \beta)$ is analytic. There is a jth-order phase transition at a boundary point of a phase if at least one $j$ th-order partial derivative of $\lambda(\rho, \beta)$ is discontinuous there, while all lower order derivatives are continuous.

7.1. First-Order Phase Transition. Recall that the Lyapunov exponent is given according to equation 72 by

$$
\lambda(\rho, \beta)=\log \left(1+e^{a}\right)-\frac{1}{\sqrt{\beta}} \int_{\log \rho}^{a} d x \sqrt{\log \frac{1+e^{a}}{1+e^{x}}},
$$

where $a$ denotes the optimal value of $h(1)$ on which the supremum of the functional $\Lambda[h(1)]$ is realized.

Hence, if $a$ is unique, we can compute that

$$
\begin{aligned}
\frac{\partial \lambda}{\partial \rho} & =\frac{e^{a}}{1+e^{a}}\left[1-\frac{1}{2 \sqrt{\beta}} \int_{\log \rho}^{a} \frac{d x}{\sqrt{\log \frac{1+e^{a}}{1+e^{x}}}}\right] \frac{\partial a}{\partial \rho}+\frac{1}{\sqrt{\beta}} \frac{1}{\rho} \sqrt{\log \frac{1+e^{a}}{1+e^{\log \rho}}} \\
& =\frac{1}{\sqrt{\beta}} \frac{1}{\rho} \sqrt{\log \left(\frac{1+e^{a}}{1+\rho}\right)}
\end{aligned}
$$


Similarly, we can compute that

$$
\frac{\partial \lambda}{\partial \beta}=\frac{1}{2 \beta^{3 / 2}} \int_{\log \rho}^{a} d x \sqrt{\log \frac{1+e^{a}}{1+e^{x}}}=\frac{1}{2 \beta}\left(-\lambda(\rho, \beta)+\log \left(1+e^{a}\right)\right) .
$$

Therefore, if $a$ is unique, the Lyapunov exponent is analytic and there is no phase transition. On the other hand, if there exist two distinct $a_{1}$ and $a_{2}$ that give the same value of $\lambda(\rho, \beta)$, then when we change the parameters $(\rho, \beta)$ continuously, the value of $a$ changes and this causes a jump discontinuity of the first-order derivative, which leads to the first-order phase transition. To summarize, if $F(a ; \rho)=2 \sqrt{\beta}$ has more than one solution that gives the same value of Lyapunov exponent, then there is a first-order phase transition. Otherwise the Lyapunov exponent is analytic in its arguments.

Proposition 16. There exists $\underline{\rho}_{c} \in(0, \infty)$ so that for any $0<\rho<\underline{\rho}_{c}$, there exists a first-order phase transition.

Proof. Let us first analyze

$$
F(a ; \rho)=\int_{\log \rho}^{a} \frac{d x}{\sqrt{\log \frac{1+e^{a}}{1+e^{x}}}} .
$$

Changing the integration variable as $y=\log \frac{1+e^{a}}{1+e^{x}}$, we get

$$
F(a ; \rho)=\int_{0}^{\log \frac{1+e^{a}}{1+\rho}} \frac{1+e^{a}}{1+e^{a}-e^{y}} \frac{1}{\sqrt{y}} d y
$$

Therefore, it suffices to study

$$
G(a)=\int_{0}^{\log \left(\frac{a}{1+\rho}\right)} \frac{a}{a-e^{y}} \frac{1}{\sqrt{y}} d y, \quad a>1+\rho
$$

which is related to $F(a ; \rho)$ as $F(a ; \rho)=G\left(1+e^{a}\right)$.

It is straightforward to compute the derivative

$$
G^{\prime}(a)=\frac{1+\rho}{a \rho} \frac{1}{\sqrt{\log \frac{a}{1+\rho}}}-\int_{0}^{\log \left(\frac{a}{1+\rho}\right)} \frac{e^{y}}{\left(a-e^{y}\right)^{2}} \frac{1}{\sqrt{y}} d y
$$

from which it follows that one has $G^{\prime}(1+\rho)=\infty$ and $G^{\prime}(\infty)=0$. 
Let $x=\frac{a}{1+\rho}$. Then, for any fixed $x>e$, using integration by parts,

$$
\begin{aligned}
G^{\prime}(a) & =\frac{1}{x \rho \sqrt{\log x}}-\int_{0}^{\log x} \frac{e^{y}}{\left((1+\rho) x-e^{y}\right)^{2}} \frac{1}{\sqrt{y}} d y \\
& \leq \frac{1}{x \rho \sqrt{\log x}}-\int_{1}^{\log x} \frac{e^{y}}{\left((1+\rho) x-e^{y}\right)^{2}} \frac{1}{\sqrt{y}} d y \\
& =\frac{1}{x \rho \sqrt{\log x}}-\int_{1}^{\log x} \frac{1}{\sqrt{y}} d\left(\frac{1}{(1+\rho) x-e^{y}}\right) \\
& =\frac{1}{x \rho \sqrt{\log x}}-\frac{1}{x \rho \sqrt{\log x}}+\frac{1}{(1+\rho) x-e}+\int_{1}^{\log x} \frac{1}{(1+\rho) x-e^{y}} d\left(\frac{1}{\sqrt{y}}\right) \\
& =\frac{1}{(1+\rho) x-e}-\frac{1}{2} \int_{1}^{\log x} \frac{1}{(1+\rho) x-e^{y}} \frac{1}{y^{3 / 2}} d y .
\end{aligned}
$$

Observe that the first term in this expression is always positive

$$
\lim _{\rho \rightarrow 0^{+}} \frac{1}{(1+\rho) x-e}=\frac{1}{x-e}
$$

while the second term diverges as $\rho \rightarrow 0_{+}$

$$
\begin{aligned}
\frac{1}{2} \int_{1}^{\log x} \frac{1}{(1+\rho) x-e^{y}} \frac{1}{y^{3 / 2}} d y & =\frac{1}{2} \int_{e}^{x} \frac{1}{(1+\rho) x-z} \frac{1}{(\log z)^{3 / 2} z} d z \\
& \geq \frac{1}{2(\log x)^{3 / 2} x} \int_{e}^{x} \frac{1}{(1+\rho) x-z} d z \\
& =\frac{1}{2(\log x)^{3 / 2} x} \log \left(\frac{(1+\rho) x-e}{\rho x}\right) \\
& \rightarrow+\infty,
\end{aligned}
$$

as $\rho \rightarrow 0^{+}$. Hence, we conclude that for any fixed $x=\frac{a}{1+\rho}>e$, the derivative $G^{\prime}(a)$ becomes negative for sufficiently small $\rho>0$

$$
\exists \underline{\rho}_{x}>0, G^{\prime}(a)<0 \text { for all } 0<\rho<\underline{\rho}_{x} .
$$

Therefore, by continuity, for any $\rho$ sufficiently small, there exists an interval on which $F^{\prime}(a ; \rho)$ is negative.

Moreover, for any $\rho>0$, the function $F(a ; \rho)$ grows without limit as $a \rightarrow \infty$. This follows from the lower bound

$$
\begin{aligned}
F(a ; \rho) & =\int_{0}^{\log \frac{1+e^{a}}{1+\rho}} \frac{1+e^{a}}{1+e^{a}-e^{y}} \frac{1}{\sqrt{y}} d y \\
& \geq \int_{0}^{\log \frac{1+e^{a}}{1+\rho}} \frac{1}{\sqrt{y}} d y=2 \sqrt{\log \frac{1+e^{a}}{1+\rho}} \rightarrow \infty,
\end{aligned}
$$

as $a \rightarrow \infty$. Hence, we conclude that $F(a ; \rho)$ is increasing on an interval $(\log \rho, \log \rho+M)$ and $F(a ; \rho)$ is decreasing on $(\log \rho+M, \log \rho+M+K)$ for 
some $M, K>0$ and $F(a ; \rho) \rightarrow \infty$ as $a \rightarrow \infty$. Hence, there is a first-order phase transition for any sufficiently small $\rho$.

Proposition 17. There exists $\bar{\rho}_{c} \in(0, \infty)$, so that for any $\rho>\bar{\rho}_{c}$, the Lyapunov exponent $\lambda(\rho, \beta)$ is analytic and hence there are no phase transitions.

Proof. Denoting $x=\frac{a}{1+\rho} \geq 1$ as above, we have

$$
\begin{aligned}
G^{\prime}(a) & =\frac{1}{x \rho \sqrt{\log x}}-\int_{0}^{\log x} \frac{e^{y}}{\left((1+\rho) x-e^{y}\right)^{2}} \frac{1}{\sqrt{y}} d y \\
& \geq \frac{1}{x \rho \sqrt{\log x}}-\frac{1}{\rho^{2} x^{2}} \int_{0}^{\log x} \frac{e^{y}}{\sqrt{y}} d y \\
& =\frac{1}{\rho x \sqrt{\log x}}\left(1-\frac{1}{\rho} H(\sqrt{\log x})\right)
\end{aligned}
$$

where we denoted

$$
H(x)=x e^{-x^{2}} \int_{0}^{x^{2}} \frac{e^{y}}{\sqrt{y}} d y=2 x e^{-x^{2}} \int_{0}^{x} d t e^{t^{2}} .
$$

The function $H(x)$ has the following properties: i) $H(0)=0$; ii) $H(x)$ is positive for $x>0$, and has a maximum at $x_{0}=1.502$ where it takes the value $H\left(x_{0}\right)=1.28475$; iii) $\lim _{x \rightarrow \infty} H(x)=1$. The property iii) follows by an application of the L'Hospital's rule

$$
\lim _{x \rightarrow \infty} \frac{2}{e^{x^{2}} \frac{1}{x}} \int_{0}^{x} d t e^{t^{2}}=\lim _{x \rightarrow \infty} \frac{2}{2-\frac{1}{x^{2}}}=1 .
$$

These properties imply that the function $H(x)$ is bounded from above as $H(x) \leq H\left(x_{0}\right)$, and thus for $\rho>H\left(x_{0}\right)$ the expression on the right-hand side of (104) is positive for any $x>1$. This shows that for sufficiently large $\rho$, there can be only one optimal $h(1)$ and thus the Lyapunov exponent $\lambda(\rho, \beta)$ is analytic.

Remark 18. Our analysis showed rigorously that there is no phase transition for sufficiently large $\rho$ and there is a first-order phase transition for sufficiently small $\rho$ but we cannot show analytically what is in between. Numerical studies in Section 5 gave strong evidence that $\bar{\rho}_{c}=\underline{\rho}_{c}$.

7.2. Second-Order Phase Transition. It has been shown in Eq. (93) that if the equation $F(a ; \rho)=2 \sqrt{\beta}$ has a unique solution for $a$, we have

$$
\frac{\partial \lambda}{\partial \rho}=\frac{1}{\sqrt{\beta}} \frac{1}{\rho} \sqrt{\log \left(\frac{1+e^{a}}{1+\rho}\right)},
$$

where $a$ is the solution of the equation $F(a ; \rho)=2 \sqrt{\beta}$.

Let us define

$$
\rho_{c}:=\sup \left\{\rho: \exists a, \frac{\partial}{\partial a} F(a ; \rho)<0\right\} .
$$


From the proof that there is no first-order phase transition for large $\rho$ and there is first-order phase transition for small $\rho$, it is easy to see that $0<$ $\rho_{c}<\infty$. Moreover, for any $\rho>\rho_{c}, \frac{\partial}{\partial a} F(a ; \rho) \geq 0$ for any $a$. By continuity, $\frac{\partial}{\partial a} F\left(a ; \rho_{c}\right) \geq 0$ for any $a$. It is also easy to see that there exists some $a_{c}$ so that $\frac{\partial}{\partial a} F\left(a_{c} ; \rho_{c}\right)=0$ and define $\beta_{c}$ as $2 \sqrt{\beta_{c}}=F\left(a_{c} ; \rho_{c}\right)$. (Note that our analysis does not show that $\beta_{c}$ is unique although numerical results suggest so.)

Proposition 19. There is a second-order phase transition at $\left(\rho_{c}, \beta_{c}\right)$. At this point all second order partial derivatives of $\lambda(\rho, \beta)$ are infinite.

Proof. First, since $\frac{\partial}{\partial a} F\left(a ; \rho_{c}\right) \geq 0$ for any $a$, by (107), there is no first-order phase transition. Next, we can compute that

$$
\frac{\partial^{2} \lambda}{\partial \rho^{2}}=\frac{1}{\sqrt{\beta} \rho \sqrt{\log \frac{1+e^{a}}{1+\rho}}}\left[-\frac{1}{\rho} \log \left(\frac{1+e^{a}}{1+\rho}\right)-\frac{1}{2(1+\rho)}+\frac{1}{2} \frac{e^{a}}{1+e^{a}} \frac{\partial a}{\partial \rho}\right],
$$

where $F(a ; \rho)=2 \sqrt{\beta}$. Off the phase transition curve, we differentiate the equation $F(a ; \rho)=2 \sqrt{\beta}$ w.r.t. $\rho$ at fixed $\beta$. Using $(93)$ we have

$$
\frac{\partial}{\partial a} F(a ; \rho) \frac{\partial a}{\partial \rho}=\frac{1}{\rho} \frac{1}{\sqrt{\log \frac{1+e^{a}}{1+\rho}}} .
$$

Since $\lim _{(\rho, \beta) \rightarrow\left(\rho_{c}, \beta_{c}\right)} \frac{\partial}{\partial a} F(a ; \rho)=0$, we have $\lim _{(\rho, \beta) \rightarrow\left(\rho_{c}, \beta_{c}\right)} \frac{\partial a}{\partial \rho}=\infty$. Moreover, since $\frac{\partial}{\partial a} F(\log \rho ; \rho)=\infty$, it is clear that at $\left(\rho_{c}, \beta_{c}\right), \log \left(\frac{1+e^{a}}{1+\rho}\right)$ does not vanish. Together, we proved that

$$
\lim _{(\rho, \beta) \rightarrow\left(\rho_{c}, \beta_{c}\right)} \frac{\partial^{2} \lambda}{\partial \rho^{2}}=\infty .
$$

Consider next the partial derivative $\partial_{\beta} \lambda$. This is given by

$$
\frac{\partial \lambda}{\partial \beta}=\frac{1}{2 \beta^{3 / 2}} \int_{\log \rho}^{a} d x \sqrt{\log \frac{1+e^{a}}{1+e^{x}}}=\frac{1}{2 \beta}\left(-\lambda(\rho, \beta)+\log \left(1+e^{a}\right)\right) .
$$

Since $\lambda(\rho, \beta)$ is continuous in its arguments, there is a first-order phase transition if $a$ has a jump discontinuity, i.e. switching from one solution to the other of the equation $F(a ; \rho)=2 \sqrt{\beta}$. It is clear that there is no first-order phase transition at $\left(\rho_{c}, \beta_{c}\right)$. Since

$$
\begin{aligned}
& \frac{\partial^{2} \lambda}{\partial \beta^{2}}=-\frac{1}{2 \beta^{2}}\left(-\lambda(\rho, \beta)+\log \left(1+e^{a}\right)\right)+\frac{1}{2 \beta}\left(-\frac{\partial \lambda}{\partial \beta}+\frac{e^{a}}{1+e^{a}} \frac{\partial a}{\partial \beta}\right) \\
& =\frac{3}{4 \beta^{2}}\left(\lambda(\rho, \beta)-\log \left(1+e^{a}\right)\right)+\frac{1}{2 \beta} \frac{e^{a}}{1+e^{a}} \frac{\partial a}{\partial \beta},
\end{aligned}
$$

and $\frac{\partial a}{\partial \beta}+\frac{1}{\sqrt{\beta}} \frac{1}{\frac{\partial}{\partial a} F(a ; \rho)} \rightarrow \infty$ as $(\rho, \beta) \rightarrow\left(\rho_{c}, \beta_{c}\right)$, there is a second-order phase transition at $\left(\rho_{c}, \beta_{c}\right)$. 
Finally we consider the cross second derivative. This can be computed in two ways, taking the derivatives in either order. We get

$$
\begin{aligned}
\frac{\partial^{2} \lambda}{\partial \rho \partial \beta} & =-\frac{1}{2 \beta^{3 / 2}} \frac{1}{\rho} \sqrt{\log \frac{1+e^{a}}{1+\rho}}+\frac{1}{\sqrt{\beta}} \frac{1}{\rho} \frac{1}{2 \sqrt{\log \frac{1+e^{a}}{1+\rho}}} \frac{e^{a}}{1+e^{a}} \frac{\partial a}{\partial \beta} \\
= & -\frac{1}{2 \beta^{3 / 2}} \frac{1}{\rho} \sqrt{\log \frac{1+e^{a}}{1+\rho}}+\frac{1}{2 \beta} \frac{e^{a}}{1+e^{a}} \frac{\partial a}{\partial \rho} .
\end{aligned}
$$

Comparing them we get the following relation between the partial derivatives of $a$, the unique solution of the variational problem,

$$
\frac{\partial a}{\partial \beta}=\frac{1}{\sqrt{\beta}} \rho \sqrt{\log \frac{1+e^{a}}{1+\rho}} \frac{\partial a}{\partial \rho} .
$$

This relation holds only off the phase transition curve, or at the critical point. Elsewhere on the phase transition curve, $a$ is discontinuous and its partial derivatives do not exist. This is an analog of the Maxwell relations, which are well-known in thermodynamics [33.

We have seen above that at the critical point $(\rho, \beta) \rightarrow\left(\rho_{c}, \beta_{c}\right)$ both partial derivatives $(\partial a / \partial \rho)$ and $(\partial a / \partial \beta)$ become infinite. This implies that the limit of $(114)$ as $(\rho, \beta) \rightarrow\left(\rho_{c}, \beta_{c}\right)$ is infinite also.

The divergence of the partial derivative $(\partial a / \partial \beta)_{\rho=\rho_{c}}$ at $\beta \rightarrow \beta_{c}$ is seen in graphical form in Figure 3. The black solid curve shows $d\left(\rho_{c}, \beta\right)$ as function of $1 / \beta$. Recalling that $d$ is related to $a$ as $(75)$, one obtains

$$
\frac{\partial d}{\partial \beta}=-\frac{1}{2 \beta} d+\frac{1}{2 \beta d}\left(1-\frac{e^{-\beta d^{2}}}{1+\rho}\right) \frac{\partial a}{\partial \beta} .
$$

This becomes infinite as we approach the critical point $\beta \rightarrow \beta_{c}$ along the curve of fixed $\rho=\rho_{c}$, as seen in Figure 3 .

\section{The Slope of the Phase Transition Curve}

We prove in this section a relation for the slope of the phase transition curve $\beta_{\mathrm{cr}}(\rho)$. This is given by the following result.

Theorem 20. (Clausius-Clapeyron relation) The slope of the phase transition curve $\beta(\rho)$ is related to the ratio of the jump discontinuities of the first partial derivatives of $\lambda(\rho, \beta)$ with respect to its arguments. This ratio is given by

$$
\frac{d \beta_{\mathrm{cr}}}{d \rho}=-\frac{\Delta\left(\frac{\partial \lambda(\rho, \beta)}{\partial \rho}\right)}{\Delta\left(\frac{\partial \lambda(\rho, \beta)}{\partial \beta}\right)}=-\frac{2}{\rho\left(d_{1}+d_{2}\right)},
$$

where the jump discontinuities of the derivatives across the phase transition curve are defined as

$$
\Delta\left(\frac{\partial \lambda(\rho, \beta)}{\partial \rho}\right)=\left.\left(\frac{\partial \lambda(\rho, \beta)}{\partial \rho}\right)\right|_{d_{2}}-\left.\left(\frac{\partial \lambda(\rho, \beta)}{\partial \rho}\right)\right|_{d_{1}},
$$


and analogous for $\Delta\left(\frac{\partial \lambda(\rho, \beta)}{\partial \beta}\right) \cdot d_{1}<d_{2}$ are the solutions of the variational problem for $\Lambda(d)$ on the phase transition curve. Recall that $d$ is related to $h(1)$ as shown in equation (75). The two solutions $d_{1,2}$ become equal at the critical point $\lim _{\rho \rightarrow \rho_{c}}\left(d_{2}-\overline{d_{1}}\right)=0$.

Proof. The proof of the relation (117) uses the continuity of the Lyapunov exponent $\lambda(\rho, \beta)$ across the phase transition line. Equating the change in $\lambda(\rho, \beta)$ as we move along the transition curve, on one side and on the other side of the curve respectively, we get

$$
\begin{aligned}
& d \lambda(\rho, \beta)=\left(\frac{\partial \lambda(\rho, \beta)}{\partial \rho}\right) d \rho+\left.\left(\frac{\partial \lambda(\rho, \beta)}{\partial \beta}\right) d \beta\right|_{\text {phase } 1} \\
& =\left(\frac{\partial \lambda(\rho, \beta)}{\partial \rho}\right) d \rho+\left.\left(\frac{\partial \lambda(\rho, \beta)}{\partial \beta}\right) d \beta\right|_{\text {phase } 2} .
\end{aligned}
$$

This gives immediately the first equality in equation (117).

In order to prove also the second equality in (117), we use the explicit expressions for the partial derivatives obtained in (93) and (94). These relations simplify when expressed in terms of the $d$ variable, related to $h(1)$ as in (75). We obtain, off the phase transition curve,

$$
\begin{aligned}
& \left(\frac{d \lambda}{d \rho}\right)=\frac{d}{\rho} \\
& \left(\frac{d \lambda}{d \beta}\right)=\frac{1}{2 \beta}\left[\beta d^{2}+\log (1+\rho)-\lambda(\rho, \beta)\right] .
\end{aligned}
$$

The jump discontinuities of these derivatives are given by

$$
\begin{aligned}
& \Delta\left(\frac{\partial \lambda(\rho, \beta)}{\partial \rho}\right)=\frac{1}{\rho}\left(d_{2}-d_{1}\right) \\
& \Delta\left(\frac{\partial \lambda(\rho, \beta)}{\partial \beta}\right)=\frac{1}{2}\left(d_{2}^{2}-d_{1}^{2}\right)
\end{aligned}
$$

Using these expressions into 117 one finds the explicit result for the slope of the phase transition curve given in the second equality of (117).

Remark 21. We note that the results of the Proposition 11 are in agreement with the relation (117) for the slope of the phase transition curve.

Remark 22. A relation of the form (117) holds also in the mean-field approximation. For this case we have to replace $d$ with a, which is the optimizer of the variational problem for $G(a ; \rho, \beta)$. The jump discontinuities of the first derivatives of $\bar{\lambda}(\rho, \beta)$ have been computed in (89) and (91), respectively, which gives the slope of the phase transition curve

$$
\frac{d \beta}{d \rho}=-\frac{\frac{1}{\rho} \Delta(\beta)}{\frac{1}{3} \Delta(\beta)}=-\frac{3}{\rho} .
$$

This agrees with the known result for the phase transition curve 85) in the mean-field approximation. 
A relation of the form (117) has been proved in [3] for the slope of the phase transition curve in the $p$-star ERGM, see Theorem 3 in [3].

\section{Critical Exponent}

We have already seen that at $\left(\rho_{c}, \beta_{c}\right), \frac{\partial}{\partial a} F\left(a_{c} ; \rho_{c}\right)=0$ and $F$ is increasing everywhere. Therefore, $\frac{\partial^{2}}{\partial a^{2}} F\left(a_{c} ; \rho_{c}\right)=0$. (Otherwise, $a_{c}$ is a local minimum (resp. local maximum) if $\frac{\partial^{2}}{\partial a^{2}} F\left(a_{c} ; \rho_{c}\right)>0$ (resp. $\left.\frac{\partial^{2}}{\partial a^{2}} F\left(a_{c} ; \rho_{c}\right)<0\right)$, which contradicts $F$ being increasing everywhere.) Moreover, since $\frac{\partial}{\partial a} F\left(a ; \rho_{c}\right)>0$ for any $a$ in a neighborhood of $a_{c}$ except at $a_{c}$ and $\frac{\partial}{\partial a} F\left(a_{c} ; \rho_{c}\right)=0$, we conclude that $a_{c}$ is a local minimum of the function $\frac{\partial}{\partial a} F\left(a ; \rho_{c}\right)$, which implies that $\frac{\partial^{3}}{\partial a^{3}} F\left(a_{c} ; \rho_{c}\right)>0$.

Along the phase transition curve, there exist $a_{1}<a_{c}<a_{2}$ such that $F\left(a_{1} ; \rho\right)=F\left(a_{2} ; \rho\right)$. If there exists the relation

$$
a_{2}-a_{1} \simeq \gamma\left|\beta-\beta_{c}\right|^{\alpha},
$$

along the phase transition curve as $\beta \rightarrow \beta_{c}$, then, the exponent $\alpha$ is called the critical exponent in statistical mechanics.

As a first step, we need to understand the asymptotic relation between $\rho-\rho_{c}$ and $\beta-\beta_{c}$ near the critical point $\left(\rho_{c}, \beta_{c}\right)$.

Along the phase transition curve,

$$
\begin{aligned}
& \log \left(1+e^{a_{1}}\right)-\frac{1}{\sqrt{\beta}} \int_{\log \rho}^{a_{1}} \sqrt{\log \frac{1+e^{a_{1}}}{1+e^{x}}} d x \\
& =\log \left(1+e^{a_{2}}\right)-\frac{1}{\sqrt{\beta}} \int_{\log \rho}^{a_{2}} \sqrt{\log \frac{1+e^{a_{2}}}{1+e^{x}}} d x .
\end{aligned}
$$

Differentiating with respect to $\rho$ and using the identity $F\left(a_{1} ; \rho\right)=F\left(a_{2} ; \rho\right)=$ $2 \sqrt{\beta}$, we get

$$
\begin{aligned}
& \frac{1}{\sqrt{\beta}} \frac{1}{\rho} \sqrt{\log \frac{1+e^{a_{1}}}{1+\rho}}+\frac{1}{2} \frac{1}{\beta^{3 / 2}} \int_{\log \rho}^{a_{1}} \sqrt{\log \frac{1+e^{a_{1}}}{1+e^{x}}} d x \frac{\partial \beta}{\partial \rho} \\
& =\frac{1}{\sqrt{\beta}} \frac{1}{\rho} \sqrt{\log \frac{1+e^{a_{2}}}{1+\rho}}+\frac{1}{2} \frac{1}{\beta^{3 / 2}} \int_{\log \rho}^{a_{2}} \sqrt{\log \frac{1+e^{a_{2}}}{1+e^{x}}} d x \frac{\partial \beta}{\partial \rho} .
\end{aligned}
$$

By 126, we can rewrite (127) as

$$
\begin{aligned}
& \frac{1}{\sqrt{\beta}} \frac{1}{\rho} \sqrt{\log \frac{1+e^{a_{1}}}{1+\rho}}+\frac{1}{2} \frac{1}{\beta} \log \left(1+e^{a_{1}}\right) \frac{\partial \beta}{\partial \rho} \\
& =\frac{1}{\sqrt{\beta}} \frac{1}{\rho} \sqrt{\log \frac{1+e^{a_{2}}}{1+\rho}}+\frac{1}{2} \frac{1}{\beta} \log \left(1+e^{a_{2}}\right) \frac{\partial \beta}{\partial \rho},
\end{aligned}
$$


which implies that

$$
\begin{aligned}
\frac{\partial \beta}{\partial \rho} & =-\frac{\frac{1}{\sqrt{\beta}} \frac{1}{\rho} \sqrt{\log \frac{1+e^{a_{2}}}{1+\rho}}-\frac{1}{\sqrt{\beta}} \frac{1}{\rho} \sqrt{\log \frac{1+e^{a_{1}}}{1+\rho}}}{\frac{1}{2} \frac{1}{\beta} \log \left(1+e^{a_{2}}\right)-\frac{1}{2} \frac{1}{\beta} \log \left(1+e^{a_{1}}\right)} \\
& \rightarrow-\frac{\sqrt{\beta_{c}}}{\rho_{c}} \frac{1}{\sqrt{\log \frac{1+e^{a_{c}}}{1+\rho_{c}}}},
\end{aligned}
$$

as $a_{2}-a_{1} \rightarrow 0$ (and thus $a_{1}, a_{2} \rightarrow a_{c},(\rho, \beta) \rightarrow\left(\rho_{c}, \beta_{c}\right)$ ).

Therefore, along the transition curve near the critical point,

$$
\beta-\beta_{c}=-\frac{\sqrt{\beta_{c}}}{\rho_{c}} \frac{1}{\sqrt{\log \frac{1+e^{a_{c}}}{1+\rho_{c}}}}\left(\rho-\rho_{c}\right)+O\left(\left(\rho-\rho_{c}\right)^{2}\right) .
$$

This agrees with the result of (117) which gives that at the critical point, the slope of the phase transition curve is

$$
\left.\frac{d \beta}{d \rho}\right|_{\rho=\rho_{c}}=-\frac{1}{\rho_{c} d_{c}}=-\frac{\sqrt{\beta_{c}}}{\rho_{c}} \frac{1}{\sqrt{\log \frac{1+e^{a_{c}}}{1+\rho}}} .
$$

Along the phase transition curve, we have

$$
F\left(a_{1} ; \rho\right)-F\left(a_{c} ; \rho_{c}\right)=2 \sqrt{\beta}-2 \sqrt{\beta_{c}} .
$$

On the one hand,

$$
\begin{aligned}
2 \sqrt{\beta}-2 \sqrt{\beta_{c}} & =\frac{1}{\sqrt{\beta_{c}}}\left(\beta-\beta_{c}\right)+O\left(\left(\beta-\beta_{c}\right)^{2}\right) \\
& =-\frac{1}{\rho_{c}} \frac{1}{\sqrt{\log \frac{1+e^{a_{c}}}{1+\rho_{c}}}}\left(\rho-\rho_{c}\right)+O\left(\left(\rho-\rho_{c}\right)^{2}\right) .
\end{aligned}
$$

On the other hand,

$$
\begin{aligned}
& F\left(a_{1} ; \rho\right)-F\left(a_{c} ; \rho_{c}\right) \\
& =\left[F\left(a_{1} ; \rho\right)-F\left(a_{1} ; \rho_{c}\right)\right]+\left[F\left(a_{1} ; \rho_{c}\right)-F\left(a_{c} ; \rho_{c}\right)\right] \\
& =-\frac{1}{\rho_{c}} \frac{1}{\sqrt{\log \frac{1+e^{a_{1}}}{1+\rho_{c}}}}\left(\rho-\rho_{c}\right)+O\left(\left(\rho-\rho_{c}\right)^{2}\right) \\
& \quad+\frac{1}{6} \frac{\partial^{3}}{\partial a^{3}} F\left(a_{c} ; \rho_{c}\right)\left(a_{1}-a_{c}\right)^{3}+O\left(\left(a_{1}-a_{c}\right)^{4}\right)
\end{aligned}
$$


Therefore, by 133 and (134,

$$
\begin{aligned}
& -\frac{1}{\rho_{c}} \frac{1}{\sqrt{\log \frac{1+e^{a_{c}}}{1+\rho_{c}}}}\left(\rho-\rho_{c}\right)+O\left(\left(\rho-\rho_{c}\right)^{2}\right) \\
& =-\frac{1}{\rho_{c}} \frac{1}{\sqrt{\log \frac{1+e^{a_{1}}}{1+\rho_{c}}}}\left(\rho-\rho_{c}\right)+O\left(\left(\rho-\rho_{c}\right)^{2}\right) \\
& +\frac{1}{6} \frac{\partial^{3}}{\partial a^{3}} F\left(a_{c} ; \rho_{c}\right)\left(a_{1}-a_{c}\right)^{3}+O\left(\left(a_{1}-a_{c}\right)^{4}\right),
\end{aligned}
$$

which implies that

$$
\begin{aligned}
& -\frac{1}{\rho_{c}}\left(\rho-\rho_{c}\right)\left(a_{1}-a_{c}\right) \frac{1}{2\left(\log \frac{1+e^{a_{c}}}{1+\rho_{c}}\right)^{3 / 2}} \frac{e^{a_{c}}}{1+e^{a_{c}}} \\
& =\frac{1}{6} \frac{\partial^{3}}{\partial a^{3}} F\left(a_{c} ; \rho_{c}\right)\left(a_{1}-a_{c}\right)^{3} \\
& \quad+O\left(\left(a-a_{c}\right)^{4}\right)+O\left(\left(\rho-\rho_{c}\right)^{2}\right)+O\left(\left|\rho-\rho_{c} \| a_{1}-a_{c}\right|\right) .
\end{aligned}
$$

Therefore, along the phase transition curve near the critical point, we have

$$
a_{1}-a_{c} \simeq-D_{c}\left|\rho-\rho_{c}\right|^{1 / 2}
$$

with

$$
D_{c}=\left(\frac{1}{\left(\log \frac{1+e^{a_{c}}}{1+\rho_{c}}\right)^{3 / 2}} \frac{3 e^{a_{c}}}{\rho_{c}\left(1+e^{a_{c}}\right)} \frac{1}{\frac{\partial^{3}}{\partial a^{3}} F\left(a_{c} ; \rho_{c}\right)}\right)^{1 / 2}
$$

Similarly,

$$
a_{2}-a_{c} \simeq D_{c}\left|\rho-\rho_{c}\right|^{1 / 2}
$$

Hence, we conclude that

$$
a_{2}-a_{1} \simeq 2 D_{c}\left|\rho-\rho_{c}\right|^{1 / 2}
$$

By (130), we also have

$$
a_{2}-a_{1} \simeq 2 D_{c}\left(\frac{\rho_{c}}{\sqrt{\beta_{c}}} \sqrt{\log \frac{1+e^{a_{c}}}{1+\rho_{c}}}\right)^{1 / 2}\left|\beta-\beta_{c}\right|^{1 / 2} .
$$

Therefore, the critical exponent is $1 / 2$.

Remark 23. We have showed that the critical exponent is $1 / 2$ which is the same as in the mean field approximation.

This result can be used to obtain the law of the approach to zero of the jump in the partial derivatives of the Lyapunov exponent near the critical point. 
Proposition 24. Near the critical point $\beta \rightarrow \beta_{c}, \rho \rightarrow \rho_{c}$, the jump discontinuities of the partial derivatives of the Lyapunov exponent approach zero as

$$
\begin{aligned}
& \Delta\left(\frac{\partial \lambda}{\partial \beta}\right)=\frac{1}{2 \beta} \log \frac{1+a_{2}}{1+a_{1}} \rightarrow c_{1}\left(\beta-\beta_{c}\right)^{1 / 2} \\
& \Delta\left(\frac{\partial \lambda}{\partial \rho}\right) \rightarrow c_{2}\left(\beta-\beta_{c}\right)^{1 / 2} .
\end{aligned}
$$

where $c_{1}, c_{2}$ are positive real constants given by

$$
\begin{aligned}
& c_{1}=\frac{2 D_{c}}{\beta_{c}\left(1+a_{c}\right)}\left(\frac{\rho_{c}}{\sqrt{\beta_{c}}} \sqrt{\log \frac{1+e^{a_{c}}}{1+\rho_{c}}}\right)^{1 / 2} \\
& c_{2}=\frac{2 D_{c}}{\beta_{c}\left(1+a_{c}\right)}\left(\frac{\rho_{c}}{\sqrt{\beta_{c}}} \sqrt{\log \frac{1+e^{a_{c}}}{1+\rho_{c}}}\right)^{-1 / 2} .
\end{aligned}
$$

Proof. Follows immediately from the relations (94) and (93) for the partial derivatives, together with the result (141) for the jump of the optimizer variables along the critical line $a_{2}-a_{1}$ near the critical point $\beta \rightarrow \beta_{c}$.

\section{Generalizations}

We consider in this Section two extensions of the results presented above: a result for the growth rate of the positive integer moments of the random variable $x_{n}$, and a generalization to the linear random recursion $x_{i+1}=$ $a_{i} x_{i}+b_{i}$ with additive i.i.d. noise $b_{i}$.

10.1. Positive integer moments. For any positive integer $q \in \mathbb{N}$ we have

$$
\begin{aligned}
\mathbb{E}\left[x_{t}^{q}\right] & =x_{0}^{q} \mathbb{E}\left[\prod_{i=0}^{n-1}\left(1+\rho e^{\sigma W_{i}-\frac{1}{2} \sigma^{2} t_{i}}\right)^{q}\right] \\
& =2^{n q} x_{0}^{q} \mathbb{E}\left[\prod_{i=0}^{n-1}\left(\frac{1}{2}+\frac{1}{2} e^{\log \rho+\sigma W_{i}-\frac{1}{2} \sigma^{2} t_{i}}\right)^{q}\right] \\
& =2^{n q} x_{0}^{q} \mathbb{E}\left[\prod_{i=0}^{n-1} e^{\left(\log \rho+\sigma W_{i}-\frac{1}{2} \sigma^{2} t_{i}\right) Y_{i}}\right],
\end{aligned}
$$

where $Y_{i}$ are i.i.d. Binomial random variables with parameters $q$ and $\frac{1}{2}$. Note that a Binomial random variable with parameters $q$ and $\frac{1}{2}$ can be written as a sum of $q$ i.i.d. Bernoulli random variables. Therefore, we can compute that for any $\theta \in \mathbb{R}$,

$$
\lim _{n \rightarrow \infty} \frac{1}{n} \log \mathbb{E}\left[e^{\theta \sum_{i=0}^{n-1} Y_{i}}\right]=q \log \left(\frac{1}{2}+\frac{1}{2} e^{\theta}\right) .
$$


By Mogulskii theorem, $\mathbb{P}\left(\frac{1}{n} \sum_{i=1}^{\lfloor n \cdot\rfloor} Y_{i} \in \cdot\right)$ satisfies a sample path large deviations principle with rate function

$$
\int_{0}^{1} I_{q}\left(g^{\prime}(x)\right) d x
$$

where $g(0)=0, g$ is absolutely continuous, $0 \leq g^{\prime} \leq q$ and the rate function is $+\infty$ otherwise and

$$
\begin{aligned}
I_{q}(x) & =\sup _{\theta \in \mathbb{R}}\left\{\theta x-q \log \left(\frac{1}{2}+\frac{1}{2} e^{\theta}\right)\right\} \\
& =q \sup _{\theta \in \mathbb{R}}\left\{\theta \frac{x}{q}-\log \left(\frac{1}{2}+\frac{1}{2} e^{\theta}\right)\right\}=q I\left(\frac{x}{q}\right) .
\end{aligned}
$$

Following the proofs for the case $q=1$, we get the following result.

Theorem 25. For any $q \in \mathbb{N}, \lambda(\rho, \beta ; q):=\lim _{n \rightarrow \infty} \frac{1}{n} \log \mathbb{E}\left[x_{n}^{q}\right]$ exists and it can be expressed in terms of a variational formula

$$
\lambda(\rho, \beta ; q)=\sup _{g \in \mathcal{G}_{q}}\left\{g(1) \log \rho+\beta \int_{0}^{1}(g(1)-g(x))^{2} d x-q \int_{0}^{1} I\left(g^{\prime}(x) / q\right) d x\right\},
$$

where $I(x)=x \log x+(1-x) \log (1-x)$ and

$\mathcal{G}_{q}:=\left\{g:[0,1] \rightarrow[0, q], g(0)=0, g\right.$ is absolutely continuous and $\left.0 \leq g^{\prime} \leq q\right\}$.

Remark 26. By replacing $g$ by $q \cdot g$ in 150 , we can express $\lambda(\rho, \beta ; q)$ as

$$
\begin{aligned}
\lambda(\rho, \beta ; q) & =\sup _{g \in \mathcal{G}}\left\{q g(1) \log \rho+q^{2} \beta \int_{0}^{1}(g(1)-g(x))^{2} d x-q \int_{0}^{1} I\left(g^{\prime}(x)\right) d x\right\} \\
& =q \lambda(\rho, q \beta ; 1) .
\end{aligned}
$$

since $\lambda \geq 0$ and $q \geq 0$, where

$\mathcal{G}=\mathcal{G}_{1}=\left\{g:[0,1] \rightarrow[0,1], g(0)=0, g\right.$ is absolutely continuous and $\left.0 \leq g^{\prime} \leq 1\right\}$.

The Euler-Lagrange equation and its solutions, the phase transitions and critical exponents for the case $q=1$ can therefore be directly applied to the general $q \in \mathbb{N}$ case.

The asymptotics (24) and (25) yield

$$
\lim _{\beta \rightarrow \infty} \frac{\lambda(\rho, \beta ; q)}{\beta}=\frac{q^{2}}{3}, \quad \text { and } \quad \lim _{\rho \rightarrow \infty}\left|\lambda(\rho, \beta ; q)-\frac{q^{2} \beta}{3}-q \log \rho\right|=0 .
$$

Also, by (24) and (152), we conclude that $\lambda(\rho, \beta ; q)$ grows quadratically in $q$ for large $q$,

$$
\lim _{q \rightarrow \infty} \frac{\lambda(\rho, \beta ; q)}{q^{2}}=\frac{\beta}{3}
$$


10.2. Lyapunov exponents for linear stochastic recursion. The results for the Lyapunov exponent can be generalized to the more general linear stochastic recursion (2) $x_{i+1}=a_{i} x_{i}+b_{i}$ where $b_{i}$ are i.i.d. positive random variables independent of $\left(a_{i}\right)_{i=0}^{\infty}$. We will show that under very mild conditions on $\left(b_{i}\right)_{i=0}^{\infty}$, we have the same Lyapunov exponent as in the $b_{i} \equiv 0$ case.

Theorem 27. For fixed $q \in \mathbb{N}$, assume that $\mathbb{E}\left[b_{0}^{q}\right]<\infty$. Then,

$$
\lim _{n \rightarrow \infty} \frac{1}{n} \log \mathbb{E}\left[\left(x_{n}\right)^{q}\right]=\lambda(\rho, \beta ; q) .
$$

Proof. Observe that

$$
\begin{aligned}
x_{n} & =a_{n-1} x_{n-1}+b_{n-1} \\
& =a_{n-1} a_{n-2} x_{n-2}+a_{n-1} b_{n-2}+b_{n-1} \\
& =a_{n-1} a_{n-2} a_{n-3} x_{n-3}+a_{n-1} a_{n-2} b_{n-3}+a_{n-1} b_{n-2}+b_{n-1} \\
& \cdots \cdots \\
& =x_{0} \prod_{i=0}^{n-1} a_{i}+b_{0} \prod_{i=1}^{n-1} a_{i}+b_{1} \prod_{i=2}^{n-1} a_{i}+\cdots+b_{n-2} a_{n-1}+b_{n-1} .
\end{aligned}
$$

Since $a_{i} \geq 1$ and $b_{i} \geq 0$ for any $i$, by Theorem 25

$$
\liminf _{n \rightarrow \infty} \frac{1}{n} \log \mathbb{E}\left[\left(x_{n}\right)^{q}\right] \geq \liminf _{n \rightarrow \infty} \frac{1}{n} \log \mathbb{E}\left[\left(x_{0} \prod_{i=0}^{n-1} a_{i}\right)^{q}\right]=\lambda(\rho, \beta ; q) .
$$

On the other hand, since $a_{i} \geq 1$, we get

$$
x_{n} \leq\left(x_{0}+b_{0}+b_{1}+\cdots+b_{n-1}\right) \prod_{i=0}^{n-1} a_{i} .
$$

Since $\left(b_{i}\right)_{i=0}^{\infty}$ and $\left(a_{i}\right)_{i=0}^{\infty}$ are independent,

$$
\mathbb{E}\left[\left(x_{n}\right)^{q}\right] \leq \mathbb{E}\left[\left(x_{0}+b_{0}+b_{1}+\cdots+b_{n-1}\right)^{q}\right] \mathbb{E}\left[\left(\prod_{i=0}^{n-1} a_{i}\right)^{q}\right]
$$

For any $q \in \mathbb{N}$, since the function $x \mapsto x^{q}$ is convex, by Jensen's inequality, we have

$$
\left(\frac{x_{0}+b_{0}+b_{1}+\cdots+b_{n-1}}{n+1}\right)^{q} \leq \frac{x_{0}^{q}+b_{0}^{q}+b_{1}^{q} \cdots+b_{n-1}^{q}}{n+1} .
$$


Hence, by Theorem 25, we conclude that

$$
\begin{aligned}
& \limsup _{n \rightarrow \infty} \frac{1}{n} \log \mathbb{E}\left[\left(x_{n}\right)^{q}\right] \\
& \leq \limsup _{n \rightarrow \infty} \frac{1}{n} \log \left(\mathbb{E}\left[\left(x_{0}+b_{0}+b_{1}+\cdots+b_{n-1}\right)^{q}\right] \mathbb{E}\left[\left(\prod_{i=0}^{n-1} a_{i}\right)^{q}\right]\right) \\
& \leq \limsup _{n \rightarrow \infty} \frac{1}{n} \log \left((n+1)^{q-1}\left[x_{0}^{q}+n \mathbb{E}\left[b_{0}^{q}\right]\right] \mathbb{E}\left[\left(\prod_{i=0}^{n-1} a_{i}\right)^{q}\right]\right) \\
& =\lambda(\rho, \beta ; q) .
\end{aligned}
$$

\section{Summary AND CONClusions}

We studied in this paper the distributional properties of a linear stochastic recursion of the form $x_{i+1}=a_{i} x_{i}+b_{i}$. The coefficients $a_{i}$ have the form $a_{i}=1+\rho e^{\sigma W_{i}-\frac{1}{2} \sigma^{2} t_{i}}$ and have Markovian dependence introduced through their dependence on a standard Brownian motion $W_{i}$. The $b_{i}$ are i.i.d. positive definite random numbers.

The main results of the paper concern the rate of growth of the positive integer moments of the variable $x_{n}$. We show that the rate of growth of these moments, or Lyapunov exponents, defined as the limit

$$
\lambda_{q}(\rho, \beta)=\lim _{n \rightarrow \infty} \frac{1}{n} \log \mathbb{E}\left[\left(x_{n}\right)^{q}\right], \quad q \in \mathbb{N},
$$

exists and is finite as $n \rightarrow \infty$, at fixed $\beta=\frac{1}{2} \sigma^{2} t_{n} n$. The function $\lambda_{q}(\rho, \beta)$ can be computed explicitly using large deviations theory and is given by the solution of a variational problem for a functional $\Lambda[f]$. We solve the variational problem and reduce it to the problem of finding the extremum of a real function defined in terms of a one-dimensional integral. The Lyapunov exponents for $q \in \mathbb{N}$ can be related to the Lyapunov exponent of the first moment $q=1$ as shown in Theorem 25 .

The solution of the variational problem shows that the Lyapunov exponents have non-analytical dependence on the parameters $(\rho, \beta)$, which is similar to a phase transition in statistical mechanics. For $\rho<\rho_{c}$, below a critical value $\rho_{c}$, the Lyapunov exponents $\lambda_{q}(\rho, \beta)$ have discontinuous derivatives along a curve in the $(\rho, \beta)$ plane, ending at a critical point $\left(\rho_{c}, \beta_{c}\right)$. Along this curve the Lyapunov exponents have a first order phase transition, and at the critical point the transition is second order.

The variational problem resulting from the application of large deviations theory has a direct physical interpretation, as the thermodynamical potential of a one-dimensional gas of particles, interacting with attractive two-body interaction energy given by the covariance function of the standard Brownian motion. The results of our paper give an exact solution of this statistical mechanics problem in the grand canonical ensemble, and 
demonstrate the presence of a phase transition in this system. The thermodynamical properties are in agreement with the solution of a lattice gas with the same interaction in the thermodynamical limit, obtained in the isobaric-isothermal ensemble [27].

As mentioned, the random multiplicative process for $x_{n}$ can be interpreted as the grand partition function of a 1-dimensional lattice gas placed in a random external field given by a standard Brownian motion. This is a lattice gas equivalent of the systems studied in [7, 10, 11 in the continuous case. Our results give the asymptotics of the moments of the grand partition function, which could be used to determine the properties of the disordered system in the quenched approximation by an application of the replica approach [20].

We note that similar moment explosions have been studied for the solutions of the diffusion and Schrödinger equations in a random medium given by the square of a Gaussian random field [4, 25].

The results of this paper have applications to the numerical simulation of stochastic differential equations. Euler discretization of certain stochastic differential equations gives linear stochastic recursions of the form considered here. The weak convergence of the discretization requires the uniform boundedness of the moments of the discretized variable, see for example [2]. Similar recursions are obtained when considering the Euler discretization of stochastic volatility models with log-normally distributed volatility. The results of this paper give explicit results for the growth rate of the moments of the stochastic variable $x_{n}$ in such models. We will present detailed application of these results and methods to models of practical interest in future work.

\section{Appendix A. Solution of the Euler-Lagrange Equation}

We present in this Appendix the solution of the Euler-Lagrange equation (69) for $h(y)$. It is useful to introduce the notation

$$
V(h)=2 \beta \log \left(1+e^{h}\right) .
$$

In terms of this function, the equation $(69)$ is written as

$$
h^{\prime \prime}(y)=-V^{\prime}(h(y)),
$$

which is analogous to Newton's law for a particle moving in the potential $V(h)$. Written in this form, it is easy to check that the following combination is a constant of motion of the equation (69)

$$
E=\frac{1}{2}\left(h^{\prime}(y)\right)^{2}+V(h(y))=V(h(1)) .
$$

The value of the constant of motion was determined from the boundary condition 70 at $y=1$ as $E=V(h(1))$. 
The relation (166) will be useful to express $h^{\prime}(y)$ in terms of $h(y)$. In particular, we can use it to write

$$
\frac{d y}{d h}=\frac{1}{\sqrt{2(E-V(h(y)))}}=\frac{1}{2 \sqrt{\beta}} \frac{1}{\sqrt{\log \frac{1+e^{h(1)}}{1+e^{h}}}} .
$$

Integrating this relation over $h$ from $h(0)$ to $h(1)$ we get an equation for $h(1)$

$$
1=\frac{1}{2 \sqrt{\beta}} \int_{h(0)=\log \rho}^{h(1)} \frac{d x}{\sqrt{\log \frac{1+e^{h(1)}}{1+e^{x}}}} .
$$

Thus we can find $h(1)$ by solving the equation

$$
F(h(1) ; \rho) \equiv \int_{\log \rho}^{h(1)} \frac{d x}{\sqrt{\log \frac{1+e^{h(1)}}{1+e^{x}}}}=2 \sqrt{\beta},
$$

Once $h(1)$ is found, the complete shape of the function $h(y)$ can be determined by solving the equation

$$
\int_{\log \rho}^{h(y)} \frac{d x}{\sqrt{\log \frac{1+e^{h(1)}}{1+e^{x}}}}=2 \sqrt{\beta} y .
$$

As the next step in the solution of the variational problem we would like to compute the functional $\Lambda[f]$ corresponding to a solution $f(x)$ of the EulerLagrange equation (62) with boundary condition (63). If the Euler-Lagrange equation has a unique solution $f(y)$, then $\lambda(\rho, \beta)=\Lambda[f]$. However, if it has several solutions, as is the case around the phase transition, the Lyapunov exponent is given by the supremum of $\Lambda[f]$ over these multiple solutions

$$
\lambda(\rho, \beta)=\sup _{f} \Lambda[f] .
$$

The main result is summarized in Proposition 10. We present here the proof of this result.

Proof. We start by expressing the functional $\Lambda[f]$ in a simpler form as (172)

$$
\Lambda[f]=\frac{1}{2} \log \rho \int_{0}^{1} d x f(x)-\frac{1}{2} \int_{0}^{1} d x f(x) \log \frac{f(x)}{1-f(x)}-\int_{0}^{1} d x \log (1-f(x)) .
$$

This is obtained by eliminating the double integral over $K(z, y)$ using the Euler-Lagrange equation (59). Multiplying this equation with $f(y)$ and integrating over $y$, this allows us to solve for the double integral. Substituting into (58) gives the result above.

There are three integrals appearing in $\Lambda[f]$. We will show next that their sum can be expressed in terms of $h(1)$ alone. We consider them in turn.

The first integral is related to $f^{\prime}(0)$ as

$$
f^{\prime}(0)=2 \beta f(0)(1-f(0)) \int_{0}^{1} d z f(z)=\frac{2 \beta \rho}{(1+\rho)^{2}} \int_{0}^{1} d z f(z) .
$$


This is obtained by taking $y=0$ in (61). This is uniquely determined by $h(1)$, as can be seen from equation (166)

$$
\frac{1}{2}\left(h^{\prime}(y)\right)^{2}+V(h(y))=V(h(1))
$$

and thus $h^{\prime}(y)=\sqrt{2(V(h(1))-V(h(y))}=2 \sqrt{\beta} \sqrt{\log \frac{1+e^{h(1)}}{1+e^{h(y)}}}$. Taking here $y=0$ we can express $h^{\prime}(0)$ in terms of $h(1)$. As a result we have

$$
I_{0}=\int_{0}^{1} d x f(x)=\frac{1}{2 \beta} h^{\prime}(0)=\frac{1}{\sqrt{\beta}} \sqrt{\log \frac{1+e^{h(1)}}{1+\rho}} .
$$

The second integral in $\Lambda[f]$ is

$$
\begin{aligned}
I_{1} & =\int_{0}^{1} d x f(x) \log \frac{f(x)}{1-f(x)}=\int_{0}^{1} d x h(x) \frac{e^{h(x)}}{1+e^{h(x)}} \\
& =-\frac{1}{2 \beta} \int_{0}^{1} d x h(x) h^{\prime \prime}(x)=\frac{1}{2 \beta}\left(\int_{0}^{1} d x\left(h^{\prime}(x)\right)^{2}+h(0) h^{\prime}(0)\right),
\end{aligned}
$$

where we integrated by parts in the last step and used $h^{\prime}(1)=0$.

Finally, the third integral is

$$
\begin{aligned}
I_{2} & =\int_{0}^{1} d x \log (1-f(x))=-\int_{0}^{1} d x \log \left(1+e^{h(x)}\right) \\
& =-\frac{1}{2 \beta} \int_{0}^{1} d x V(h(x))=-\frac{1}{2 \beta} \int_{0}^{1} d y\left(E-\frac{1}{2}\left(h^{\prime}(y)\right)^{2}\right) \\
& =-\log \left(1+e^{h(1)}\right)+\frac{1}{4 \beta} \int_{0}^{1} d y\left(h^{\prime}(y)\right)^{2},
\end{aligned}
$$

where $V(h)$ is defined in (164). In the second line we used 166) to eliminate $V(h(y))$ in terms of $\left(h^{\prime}(y)\right)^{2}=4 \beta \log \frac{1+e^{h(1)}}{1+e^{h(y)}}$.

The integrals $I_{1}$ and $I_{2}$ appear in $\Lambda[f]$ in the combination

$$
\begin{aligned}
\frac{1}{2} I_{1}+I_{2} & =\frac{1}{2 \beta} \int_{0}^{1} d x\left\{\frac{1}{2}\left(h^{\prime}(x)\right)^{2}-V(h(x))\right\}+\frac{1}{4 \beta} h(0) h^{\prime}(0) \\
& =\frac{1}{\sqrt{\beta}} \int_{\log \rho}^{h(1)} d x \sqrt{\log \frac{1+e^{h(1)}}{1+e^{x}}}-\log \left(1+e^{h(1)}\right)+\frac{1}{4 \beta} \log \rho h^{\prime}(0) .
\end{aligned}
$$

Substituting this into 172 the last term cancels against the term proportional to the integral $I_{0}$, and we obtain the result $(71)$. This concludes the proof of (71). 


\section{Appendix B. Analytical solution for $\beta d^{2} \gg 1$}

We present in this Appendix an analytical solution of the variational problem for $\Lambda(d)$ in the $\beta d^{2} \rightarrow \infty$ limit. This is used to derive the properties of the phase transition in the same limit, which are summarized in Proposition 11 .

We will prove here the following approximation for the functional $\Lambda(d)$

$$
\Lambda(d)=\beta d^{2}-\frac{2}{3} \beta d^{3}+d \log \left(\frac{\rho}{1+\rho}\right)+\log (1+\rho)+o\left(a^{-1}\right),
$$

where $a=\beta d^{2}$.

The starting point is the observation that the integral appearing in 76 depends only on the combination $a=\beta d^{2}$. We denote it as

$$
J(a ; \rho)=\int_{0}^{1} \frac{y^{2} d y}{1+\rho-e^{a\left(y^{2}-1\right)}}
$$

We would like to obtain an approximation for this integral for $a \gg 1$. This is given by the following result.

Lemma 28. The integral $J(a ; \rho)$ has the following expansion for $a \gg 1$

$$
J(a ; \rho)=\frac{1}{1+\rho}\left\{\frac{1}{3}-\frac{1}{2 a} \log \left(\frac{\rho}{1+\rho}\right)+o\left(a^{-2}\right)\right\} .
$$

Proof. This result is shown by proving matching lower and upper bounds for the integral $J(a ; \rho)$. We start by deriving a lower bound for the integral. This bound follows from using the inequality $y^{2}-1 \geq 2(y-1)$, which holds for any $y \in(0,1)$, in the exponent in the denominator of the integral 180 . Then the integral can be performed exactly with the result

$$
\begin{aligned}
J(a ; \rho) & \geq \frac{1}{3 a^{3}(1+\rho)}\left\{a^{3}-\frac{3}{2} a^{2} \log \left(\frac{\rho}{1+\rho}\right)\right. \\
& \left.-\frac{3}{2} a \operatorname{Li}_{2}\left(\frac{1}{1+\rho}\right)+\frac{3}{4} \operatorname{Li}_{3}\left(\frac{1}{1+\rho}\right)-\frac{3}{4} \operatorname{Li}_{3}\left(\frac{e^{-2 a}}{1+\rho}\right)\right\}
\end{aligned}
$$

Here $\operatorname{Li}_{n}(z)$ denotes the polylogarithm of order $n[1$.

For $\rho \rightarrow 0$ and $a \gg 1$ the polylogarithms approach constant values

$$
\begin{aligned}
& \lim _{\rho \rightarrow 0} \operatorname{Li}_{2}\left(\frac{1}{1+\rho}\right)=\frac{\pi^{2}}{6} \\
& \lim _{\rho \rightarrow 0} \operatorname{Li}_{3}\left(\frac{1}{1+\rho}\right)=\zeta(3) \simeq 1.202 \\
& \lim _{x \rightarrow 0} \operatorname{Li}_{3}(x)=x+o\left(x^{2}\right) .
\end{aligned}
$$

We get thus the lower bound

$$
J(a ; \rho) \geq \frac{1}{3(1+\rho)}-\frac{1}{2 a(1+\rho)} \log \left(\frac{\rho}{1+\rho}\right)+o\left(a^{-2}\right) .
$$


Next we prove the upper bound for the integral $J(a ; \rho)$

$$
J(a ; \rho) \leq \frac{1}{1+\rho}\left\{\frac{1}{3}-\frac{1}{2 a} \log \left(\frac{\rho}{1+\rho-e^{-a}}\right)\right\} .
$$

This is obtained by considering the difference

$$
\frac{1}{1+\rho-e^{a\left(y^{2}-1\right)}}-\frac{1}{1+\rho}=\frac{1}{(1+\rho)\left[(1+\rho) e^{a\left(1-y^{2}\right)}-1\right]}
$$

Multiplying with $y^{2}$ and integrating over $y:(0,1)$ we have

$$
\begin{aligned}
& J(a ; \rho)-\frac{1}{3(1+\rho)} \leq \frac{1}{1+\rho} \int_{0}^{1} \frac{y d y}{(1+\rho) e^{a\left(1-y^{2}\right)}-1} \\
& =-\frac{1}{2 a(1+\rho)} \log \left(\frac{\rho}{1+\rho-e^{-a}}\right)<-\frac{1}{2 a(1+\rho)} \log \left(\frac{\rho}{1+\rho}\right) .
\end{aligned}
$$

This proves the upper bound (187). Comparing with the lower bound 186 we obtain the expansion (181).

The final result 179 follows directly from using the expansion (181) of the integral $J(a ; \rho)$ into the expression for $\Lambda(d)$.

We use next the approximation 179 to study the solution of the variational problem for $\Lambda(d)$ for $\beta \rightarrow \infty$. We would like to find the supremum over $d$ of the cubic polynomial in $(179)$. This supremum is reached at $d=0$ or $d_{*}=\frac{1}{2}\left(1+\sqrt{1+\frac{2}{\beta} \log \frac{\rho}{1+\rho}}\right)>0$. according to the following condition

$$
\sup _{d} \Lambda(d)=\left\{\begin{array}{cl}
\Lambda(0)=\log (1+\rho) & \text { if } \beta \leq-\frac{8}{3} \log \frac{\rho}{1+\rho} \\
\Lambda\left(d_{*}\right)>\log (1+\rho) & \text { if } \beta>-\frac{8}{3} \log \frac{\rho}{1+\rho}
\end{array}\right.
$$

The supremum switches branches at the point

$$
\beta_{\mathrm{cr}}(\rho)=-\frac{8}{3} \log \left(\frac{\rho}{1+\rho}\right)
$$

which thus defines the phase transition curve for $\beta \rightarrow \infty$. This proves the result (79). As $\beta \rightarrow \infty$ or equivalently $\rho \rightarrow 0$, the slope of this curve in $(-\log \rho, \beta)$ coordinates approaches the value $8 / 3$. This is smaller than the slope of the phase transition curve in the mean-field approximation, which is equal to 3 .

The values of $d_{1}, d_{2}$ along the phase transition curve approach

$$
d_{1}=0, \quad d_{2}=\frac{3}{4}=0.75
$$

as $\beta \rightarrow \infty$. This proves the result $(78)$. For $\beta \gg \beta_{\text {cr }}(\rho)$, we have $\lim _{\beta \rightarrow \infty} d=$ $1_{-}$and the Lyapunov exponent becomes

$$
\lim _{\beta \rightarrow \infty} \lambda(\rho, \beta)=\lim _{\beta \rightarrow \infty} \Lambda(d)=\frac{1}{3} \beta+\log \rho .
$$

This result is in agreement with the large $\beta$ asymptotic behavior of the Lyapunov exponent proven in Proposition 2, see Eq. (24). 


\section{ACKNOWLEDGEMEnTS}

We would like to thank the Editor and two anonymous referees for useful comments and advice.

\section{REFERENCES}

[1] M. Abramowitz and I. A. Stegun. Handbook of Mathematical Functions with Formulas, Graphs and Mathematical Tables. Dover Publications, New York (1972).

[2] A. Alfonsi, High order discretization schemes for the CIR process: application to Affine Term Structure and Heston models. Math. Comp. 79, 209-237 (2010).

[3] D. Aristoff and L. Zhu. On the phase transition curve in a directed exponential random graph model. arXiv:1404.6514 math.PR]. (2014).

[4] A. Asselah, P. Dai Pra, J. L. Lebowitz and Ph. Mounaix, Diffusion effects on the breakdown of a linear amplifier model driven by the square of a Gaussian field. J. Stat. Phys. 104, 24-32 (1990).

[5] F. Black, E. Derman and W. Toy. A one-factor model of interest rates and its application to treasury bond options. Financial Analysts Journal 46, 24-32 (1990).

[6] J. P. Bouchaud and A. Georges, Anomalous diffusion in disordered media: Statistical mechanisms, models and physical applications. Physics Reports 195, 127-293 (1990).

[7] K. Broderix and R. Kree. Thermal equilibrium with the Wiener potential: Testing the replica variational approximation. Europhys. Lett. 32, 343 (1995)

[8] S. Chatterjee and P. Diaconis. Estimating and understanding exponential random graph models. Annals of Statistics. 41, 2428-2461 (2013).

[9] J. E. Cohen and C. M. Newman. The stability of large random matrices and their products. The Annals of Probability. 12, 283-310 (1984).

[10] A. Comtet and C. Monthus. On the flux distribution in a one-dimensional disordered system. J. Phys. I 4, 635 (1994)

[11] A. Comtet, C. Monthus and M. Yor. Exponential functionals of Brownian motion and disordered systems. J. Appl. Prob. 35, 255-271 (1998)

[12] A. Dembo and O. Zeitouni. Large Deviations Techniques and Applications, 2nd Edition, Springer, New York, 1998.

[13] D. Dufresne. The integral of geometric Brownian motion. Adv. Appl. Prob.. 33, 223241 (2001).

[14] D. Dufresne. The log-normal approximation in financial and other computations. Adv. Appl. Prob.. 36, 747-773 (2004).

[15] R. Ellis. Entropy, Large Deviations, and Statistical Mechanics (Classics in Mathematics). Springer, New York, 2005.

[16] C. M. Goldie. Implicit renewal theory and tails of solutions of random equations. Ann. Appl. Prob. 1, 126 (1991).

[17] L. P. Kadanoff. Statistical Physics: Statics, Dynamics and Renormalization. World Scientific, Singapore, 2000.

[18] H. Kesten. Random difference equations and renewal theory for products of random matrices. Acta Math. 131, 207 (1973).

[19] J. Messer and H. Spohn. Statistical Mechanics of the Isothermal Lane-Emden Equation. J. Stat. Phys. 29, 561 (1982).

[20] M. Mèzard, G. Parisi and M. Virasoro, Spin glass theory and beyond. Singapore, World Scientific (1987).

[21] J. Lebowitz and O. Penrose. Rigorous treatment of the van der Waals-Maxwell theory of the liquid-vapor transition. J. Math. Phys. 7, 98 (1966).

[22] R. C. Lewontin and D. Cohen. On population growth in a randomly varying environment. Proc. Natl. Acad. Sci. USA 62, 1056 (1969). 
[23] T. Mikosh, G. Samorodnitsky and L. Tafakori. Fractional moments of solutions to stochastic recurrence equations. J. Appl. Prob. 50, 969-982 (2013).

[24] M. Mitzenmacher. A brief history of generative models for power law and log-normal distributions. Internet Math. 1, 226-251 (2004).

[25] P. Mounaix, P. Collet and J. L. Lebowitz, Propagation Effects on the Breakdown of a Linear Amplifier Model: Complex-Mass Schrödinger Equation Driven by the Square of a Gaussian Field. Comm. Math. Phys. 264, 741-758 (2006).

[26] D. Pirjol. Emergence of heavy tailed distributions in a random multiplicative model driven by a Gaussian stochastic process. J. Stat. Phys. 154, 781-806 (2014).

[27] D. Pirjol. Long term growth rate in a random multiplicative model. J. Math. Phys. 55, 083305 (2014).

[28] C. Radin and M. Yin. Phase transitions in exponential random graphs. Annals of Applied Probability. 23, 2458-2471 (2013).

[29] D. Ruelle. Analyticity properties of the characteristic exponents of random matrix products. Adv. Math. 32 68-80 (1979).

[30] A. Roitershtein. One-dimensional linear recursions with Markov-dependent coefficients. Ann. Appl. Prob. 17, 572 (2007).

[31] B. DeSaporta. Tail of the stationary solution of the stochastic equation $Y_{n+1}=$ $a_{n} Y_{n}+b_{n}$ with Markovian coefficients. Stoch. Proc. Appl.. 115, 1954 (2005).

[32] D. Sornette and R. Cont. Convergent multiplicative processes repelled from zero: power laws and truncated power laws. J. Phys. I 7, 431 (1997).

[33] E. H. Stanley. Introduction to Phase Transitions and Critical Phenomena. Oxford University Press, 1987.

[34] S. R. S. Varadhan. Large Deviations and Applications, SIAM, Philadelphia, 1984.

[35] W. Vervaat. On a stochastic difference equation and a representation of nonnegative infinitely divisible random variables. Adv. Appl. Prob. 11, 750-783 (1979).

[36] M. Yor. On some exponential functions of Brownian motion. Adv. Appl. Prob. 24, 509-531 (1992).

E-mail address: dpirjol@gmail.com

E-mail address: zhul@umn.edu 